\title{
REALIZATION THEORY FOR RATIONAL SYSTEMS: THE EXISTENCE OF RATIONAL REALIZATIONS*
}

\author{
JANA NĚMCOVÁ ${ }^{\dagger}$ AND JAN H. VAN SCHUPPEN ${ }^{\dagger}$
}

\begin{abstract}
In this paper we solve the problem of the existence of rational realizations of response maps. Sufficient and necessary conditions for a response map to be realizable by a rational system are presented. We provide also the characterization of the existence of rationally observable and canonical rational realizations for a given response map.
\end{abstract}

Key words. rational systems, realization theory, algebraic reachability, rational observability

AMS subject classifications. 93B15, 93B27, 93C10

DOI. $10.1137 / 080714506$

1. Introduction. The motivation to investigate realization theory of rational systems is the use of rational systems as models of phenomena in life sciences, in particular, in systems biology. For example, rational systems occur as models of metabolic, genetic, and signaling networks. They can be found also in engineering, physics, and economics. Moreover, as Bartosiewicz stated in [3], the theory of rational systems could be simpler and more powerful, once it is developed, than the theory of smooth systems.

The realization problem for rational systems considers a map from input functions to output functions and asks whether there exists a finite-dimensional rational system with an initial condition such that its input/output map is identical to the considered map. Such a system is then called a realization of the considered input/output map. A generalization, which is not addressed in this paper, is to regard any relation between observed variables and ask for a realization as a rational system. Another goal of realization theory is to characterize certain properties of realizations. One wants to find the conditions under which the systems realizing the considered map are observable, controllable, or minimal. The relations between realizations having these properties are also of interest, since they can be applied in control and observer synthesis and in system identification.

Polynomial and rational systems are a special class of nonlinear systems admitting a more refined algebraic structure. Realization theory for discrete-time polynomial systems was formulated by Sontag in [16]. Later, in [18], Wang and Sontag published their results on realization theory for polynomial and rational continuous-time systems based on the approach of formal power series in noncommuting variables and on the relation of two characterizations of observation spaces.

Another approach to realization theory for polynomial continuous-time systems, motivated by the results of Jakubczyk in [11] for nonlinear realizations, is introduced by Bartosiewicz in [1, 4]. This approach is based on [16]. Furthermore, in [3], Bartosiewicz introduces the concept of rational systems and deals with the problem of immersion of smooth systems into rational systems. Since this problem is similar to

\footnotetext{
${ }^{*}$ Received by the editors January 28, 2008; accepted for publication July 17, 2009; published electronically October 14, 2009.

http://www.siam.org/journals/sicon/48-4/71450.html

${ }^{\dagger}$ Centrum voor Wiskunde en Informatica, Science Park 123, Amsterdam, 1098 XG, The Netherlands (J.Nemcova@cwi.nl, J.H.van.Schuppen@cwi.nl).
} 
the problem of rational realization, there is an analogy between our approach to the realization theory for rational systems and Bartosiewicz's results presented in $[3,4]$.

Compared to the realization theory for rational systems developed by Wang and Sontag in [18], our approach is different. We apply the algebraic-geometric approach rather than techniques based on formal power series. We solve the same problem of existence of rational realizations (compare Theorem 5.2 in [18] and Theorem 5.16 in this paper). In addition we deal with the questions of rational observability and algebraic reachability of rational realizations which are not treated in [18]. Another major difference is that the realizations within the class of rational systems which we consider do not have to be affine in the inputs as assumed by Wang and Sontag. This is motivated by the planned application of realization theory to biochemical systems where the inputs may enter in a rational way.

The first step, motivated by biochemical reaction networks in developing a realization theory for rational positive systems is done in [17]. We leave the problem of rational realization with the positivity constraint for further research.

The organization of the paper is as follows. Terminology, notation, and mathematical preliminaries are provided in section 2. Section 3 introduces the concept of rational systems, and section 4 introduces the classes of admissible inputs which are considered domains for response maps. The problem of rational realization is formulated, and necessary and sufficient conditions for the existence of a rational realization of a response map are presented in section 5. Section 6 deals with canonical rational realizations. Section 7 concludes the paper.

2. Algebraic preliminaries. In this section we introduce algebraic-geometric framework in which we formulate the problem of rational realization. Note that the methods of algebraic geometry are already known for control and system theory; see, for example, $[6,8,9]$. For the basic definitions and theorems of commutative algebra and algebraic geometry, see [5, 7, 12, 13, 19].

By a polynomial in finitely many indeterminates $X_{1}, \ldots, X_{n}$ with real coefficients, we mean a sum $\sum_{k \in \mathbb{N}^{n}} c(k) \prod_{i=1}^{n} X_{i}^{k(i)}$ where only finitely many coefficients $c(k) \in \mathbb{R}$ are nonzero. We denote the ring of all polynomials in $n$ variables with real coefficients by $\mathbb{R}\left[X_{1}, \ldots, X_{n}\right]$. Because the field $\mathbb{R}$ is an integral domain, so is the algebra $\mathbb{R}\left[X_{1}, \ldots, X_{n}\right]$. Therefore, we can define the field of quotients of $\mathbb{R}\left[X_{1}, \ldots, X_{n}\right]$ as the set of fractions $\left\{p / q \mid p, q \in \mathbb{R}\left[X_{1}, \ldots, X_{n}\right], q \neq 0\right\}$. This field is denoted by $\mathbb{R}\left(X_{1}, \ldots, X_{n}\right)$, and we refer to its elements as rational functions. Generally, we use the notation $\mathcal{Q}(S)$ for the field of quotients of an integral domain $S$. For example, $\mathcal{Q}\left(\mathbb{R}\left[X_{1}, \ldots, X_{n}\right]\right)=\mathbb{R}\left(X_{1}, \ldots, X_{n}\right)$. By $R / I$, for a ring $R$ and an ideal $I \subseteq R$, we denote the quotient (factor) ring of $R$ modulo $I$.

Polynomial and rational functions on varieties. A real affine variety $X$ is an algebraic variety in $\mathbb{R}^{n}$, i.e., there are finitely many $f_{1}, \ldots, f_{N} \in \mathbb{R}\left[X_{1}, \ldots, X_{n}\right]$ such that $X=\left\{\left(x_{1}, \ldots, x_{n}\right) \in \mathbb{R}^{n} \mid f_{1}\left(x_{1}, \ldots, x_{n}\right)=\cdots=f_{N}\left(x_{1}, \ldots, x_{n}\right)=0\right\}$. We say that a variety is irreducible if we cannot write it as an union of two nonempty varieties which are its strict subvarieties. Let $I \subseteq \mathbb{R}\left[X_{1}, \ldots, X_{n}\right]$ be the ideal of all polynomials which are zero at every point of the variety $X$. By a polynomial on a variety $X$, we mean a map $p: X \rightarrow \mathbb{R}$ for which there exists $q \in \mathbb{R}\left[X_{1}, \ldots, X_{n}\right]$ such that $p=q$ on $X$. The algebra of all polynomials on $X$, denoted by $A$, is then isomorphic to $\mathbb{R}\left[X_{1}, \ldots, X_{n}\right] / I$. From Hilbert basis theorem and from the fact that the ideals in the quotient ring $\mathbb{R}\left[X_{1}, \ldots, X_{n}\right] / I$ are in one-to-one correspondence with the ideals of $\mathbb{R}\left[X_{1}, \ldots, X_{n}\right]$ containing $I$, every ideal in $\mathbb{R}\left[X_{1}, \ldots, X_{n}\right] / I$ is finitely 
generated. Therefore, $A$ is a finitely generated algebra of polynomials. Since $X$ is an irreducible variety, $A$ is an integral domain. Therefore, we can define the field $Q$ of quotients of $A$. The elements of $Q$ are called rational functions on $X$.

On $\mathbb{R}^{n}$ we consider the Zariski topology, which is a topology where the closed sets are defined as real affine varieties. A variety $X \subseteq \mathbb{R}^{n}$ is endowed with the related topology, the Zariski topology on $X$. We refer to an open/closed/dense set in Zariski topology as to $Z$-open $/ Z$-closed/ $Z$-dense set. More details can be found in $[10,12]$.

Rational vector fields. Let $X$ be an irreducible real affine variety, let $A$ be the algebra of polynomials on $X$, and let $Q$ denote the field of rational functions on $X$.

Definition 2.1. A rational vector field $f$ on $X$ is an $\mathbb{R}$-linear map $f: Q \longrightarrow Q$ such that $f(\varphi \cdot \psi)=f(\varphi) \cdot \psi+\varphi \cdot f(\psi)$ for $\varphi, \psi \in Q$.

We say that the rational vector field $f$ is defined at the point $x \in X$ if $f\left(O_{x}\right) \subseteq O_{x}$, where $O_{x}=\{\varphi \in Q \mid \varphi$ is defined at $x\}$. The set $X(f)$ of all points at which a rational vector field $f$ is defined is given as $X(f)=\left\{x \in X \mid f\left(O_{x}\right) \subseteq O_{x}\right\}$.

Definition 2.2. The trajectory of a rational vector field $f$ from a point $x_{0} \in$ $X(f)$ is the map $x:[0, T) \rightarrow X(f) \subseteq X$ such that for $t \in[0, T)$ and $\varphi \in A$

$$
\frac{d}{d t}(\varphi \circ x)(t)=(f \varphi)(x(t)) \text { and } x(0)=x_{0} .
$$

Note that it is sufficient to consider only the polynomials $\varphi \in A$ in the definition above, as it is proved in [3].

Theorem 2.3 (see [3], Theorem 1). For any rational vector field $f$ and any point $x_{0} \in X(f)$, there exists a unique trajectory of $f$ from $x_{0}$ defined on the maximal interval $[0, T)$ (T may be infinite).

Proof. The proof of this statement can be found in $[2,3]$.

3. Rational systems. We consider rational systems as systems on irreducible real affine varieties with the dynamics defined by rational vector fields and with output functions having rational components. This concept of rational systems is introduced in [3], and we recall it in this section.

We consider an input space $U$ to be an arbitrary set $U \subseteq \mathbb{R}^{m}$. As an output space, we consider $\mathbb{R}^{r}$. By a slight modification of [3, Definition 2], we define rational systems as follows.

Definition 3.1. A rational system $\Sigma$ with an input space $U$ and an output space $\mathbb{R}^{r}$ is a quadruple $\Sigma=\left(X, f, h, x_{0}\right)$, where

(i) $X$ is an irreducible real affine variety;

(ii) $f=\left\{f_{\alpha} \mid \alpha \in U\right\}$ is a family of rational vector fields on $X$;

(iii) $h: X \rightarrow \mathbb{R}^{r}$ is an output map with rational components, i.e., $h_{i} \in Q$ for $i=1, \ldots, r$;

(iv) $x_{0} \in X$ is an initial state such that all $h_{i}, i=1, \ldots, r$ and all $f_{\alpha}, \alpha \in U$ are defined at $x_{0}$.

The states at which all components of the output function $h$ are defined and at which at least one of the rational vector fields $f_{\alpha}, \alpha \in U$ is defined is a $Z$-dense open subset of $X$; see [3]. If $U$ is a finite set, then the set of points at which all components of $h$ and all vector fields $f_{\alpha}, \alpha \in U$ are defined is also a $Z$-dense open subset of $X$.

As the space of input functions, we consider the set $\mathcal{U}_{p c}$ of piecewise-constant functions $u:[0, \infty] \rightarrow U$. Let $u \in \mathcal{U}_{p c}$; then $u=\left(\alpha_{1}, t_{1}\right) \ldots\left(\alpha_{n_{u}}, t_{n_{u}}\right)$ means that for $t \in\left(\sum_{j=0}^{i} t_{j}, \sum_{j=0}^{i+1} t_{j}\right]$, the input $u(t)=\alpha_{i+1} \in U$ for $i=0,1, \ldots, n_{u}-1, t_{0}=0$, and $u(0)=\alpha_{1}$. Every input $u \in \mathcal{U}_{p c}$ has a time domain $\left[0, T_{u}\right]$, where $T_{u}=\sum_{j=1}^{n_{u}} t_{j}$. If $u=$ 
$\left(\alpha_{1}, t_{1}\right) \ldots\left(\alpha_{n}, t_{n}\right), v=\left(\beta_{1}, s_{1}\right) \ldots\left(\beta_{k}, s_{k}\right) \in \mathcal{U}_{p c}$, then $(u)(v) \in \mathcal{U}_{p c}$ is an input which we get by concatenating $v$ to $u$, i.e., $(u)(v)=\left(\alpha_{1}, t_{1}\right) \ldots\left(\alpha_{n}, t_{n}\right)\left(\beta_{1}, s_{1}\right) \ldots\left(\beta_{k}, s_{k}\right)$. To express that the input $u$ was applied only on time domain $[0, t] \subseteq\left[0, T_{u}\right]$, we write a subindex $[0, t]$ to $u$ like $u_{[0, t]}$. The empty input $e$ is such input that $T_{e}=0$.

Consider a rational system $\Sigma=\left(X, f, h, x_{0}\right)$. The trajectory of the system $\Sigma$ corresponding to a constant input $u=\left(\alpha, T_{u}\right) \in \mathcal{U}_{p c}$ is the trajectory of the rational vector field $f_{\alpha}$ from $x_{0}$, i.e., it is the map $x\left(\cdot ; x_{0}, u\right):\left[0, T_{u}\right] \rightarrow X$ for which $\frac{d}{d t}(\varphi \circ x)\left(t ; x_{0}, u\right)=\left(f_{\alpha} \varphi\right)\left(x\left(t ; x_{0}, u\right)\right)$ and $x\left(0 ; x_{0}, u\right)=x_{0}$ for $t \in\left[0, T_{u}\right]$ and for $\varphi \in A$. Note that a trajectory of a vector field on a closed interval is defined as in Definition 2.2; see [4]. The trajectory of the system $\Sigma$ corresponding to an input $u=\left(\alpha_{1}, t_{1}\right) \ldots\left(\alpha_{n_{u}}, t_{n_{u}}\right) \in \mathcal{U}_{p c}$ with $T_{u}=\sum_{j=1}^{n_{u}} t_{j}$ is the map $x\left(\cdot ; x_{0}, u\right):\left[0, T_{u}\right] \rightarrow X$ such that $x\left(0 ; x_{0}, u\right)=x_{0}$, and $x\left(t ; x_{0}, u\right)=x_{\alpha_{i}}\left(t-\sum_{j=0}^{i-1} t_{j}\right)$ for $t \in\left[\sum_{j=0}^{i-1} t_{j}, \sum_{j=0}^{i} t_{j}\right]$, $t_{0}=0, i=1, \ldots, n_{u}$, where $x_{\alpha_{i}}:\left[0, t_{i}\right] \rightarrow X$ is a trajectory of a vector field $f_{\alpha_{i}}$ from the initial state $x\left(\sum_{j=0}^{i-1} t_{j} ; x_{0}, u\right)=x_{\alpha_{i-1}}\left(t_{i-1}\right)$ for $i=2, \ldots, n_{u}$ and from the initial state $x_{0}$ for $i=1$. Since a trajectory of a rational system $\Sigma$ does not need to exist for every input $u \in \mathcal{U}_{p c}$ (see [3]), we define the set of admissible inputs for $\Sigma$.

Definition 3.2. Let $\Sigma=\left(X, f, h, x_{0}\right)$ be a rational system. We define the set of admissible inputs $\mathcal{U}_{p c}(\Sigma)$ for the system $\Sigma$ as a subset of the set of piecewise-constant inputs $\mathcal{U}_{p c}$ for which there exist a trajectory of $\Sigma$.

Note that for every $u \in \mathcal{U}_{p c}(\Sigma)$ it holds that $u_{[0, t]} \in \mathcal{U}_{p c}(\Sigma)$ for every $t \in\left[0, T_{u}\right]$. We conclude the convention that a trajectory of a rational system $\Sigma$ with an initial state $x_{0} \in X$ is for the empty input $e$ equal to $x_{0}$, i.e., $x\left(0 ; x_{0}, e\right)=x_{0}$. The set of admissible inputs $\mathcal{U}_{p c}(\Sigma)$ may contain only the empty input $e$.

Remark 3.3. Every input of $\mathcal{U}_{p c}$ has finitely many switching time-points. This property is needed for the proof of irreducibility of the smallest variety containing a reachable set of a rational system (see Proposition 5.5), which is used in the proof of Theorem 6.1. It is also a necessary condition for proving that the set of functions analytic at the switching time-points of such inputs is an integral domain (see Theorem 4.4). This is the crucial property of a class of functions for which there exists a rational realization.

4. Admissible inputs. Let $U \subseteq \mathbb{R}^{m}$ be an input space. We define the sets of admissible inputs for rational systems with the values in $U$. These sets of inputs are the sets on which the response maps studied with respect to the realization problem for rational systems are defined.

Definition 4.1. A set $\widehat{\mathcal{U}_{p c}} \subseteq \mathcal{U}_{p c}$ of input functions with the values in an input space $U \subseteq \mathbb{R}^{m}$ is called a set of admissible inputs if

(i) $\forall u \in \widetilde{\mathcal{U}_{p c}} \forall t \in\left[0, T_{u}\right]: u_{[0, t]} \in \widetilde{\mathcal{U}_{p c}}$,

(ii) $\forall u \in \widetilde{\mathcal{U}_{p c}} \forall \alpha \in U \exists t>0:(u)(\alpha, t) \in \widetilde{\mathcal{U}_{p c}}$,

(iii) $\forall u=\left(\alpha_{1}, t_{1}\right) \ldots\left(\alpha_{k}, t_{k}\right) \in \widetilde{\mathcal{U}_{p c}} \exists \delta>0 \forall \overline{t_{i}} \in\left[0, t_{i}+\delta\right], i=1, \ldots, k$ :

$$
\bar{u}=\left(\alpha_{1}, \overline{t_{1}}\right) \ldots\left(\alpha_{k}, \overline{t_{k}}\right) \in \widetilde{\mathcal{U}_{p c}} .
$$

Definition 4.2. Consider a set $\widetilde{\mathcal{U}_{p c}}$ of admissible inputs with the values in $U \subseteq \mathbb{R}^{m}$. Let $u \in \widetilde{\mathcal{U}_{p c}}$. We denote the derivation of a real function $\varphi: \widetilde{\mathcal{U}_{p c}} \rightarrow \mathbb{R}$ at the switching time-point $T_{u}$ of the input $(u)(\alpha, t) \in \widetilde{\mathcal{U}_{p c}}$, where $t>0$ is sufficiently small and $\alpha \in U$ as

$$
\left(D_{\alpha} \varphi\right)(u)=\left.\frac{d}{d t} \varphi((u)(\alpha, t))\right|_{t=0+}
$$

Copyright (c) by SIAM. Unauthorized reproduction of this article is prohibited. 
Let $\varphi: \widetilde{\mathcal{U}_{p c}} \rightarrow \mathbb{R}$ be a real function, and let $u=\left(\alpha_{1}, t_{1}\right) \ldots\left(\alpha_{k}, t_{k}\right) \in \widetilde{\mathcal{U}_{p c}}$ for a set $\widetilde{\mathcal{U}_{p c}}$ of admissible inputs. Consider the function $\widehat{\varphi}_{u}(t)=\varphi\left(u_{[0, t]}\right)$ defined for $t \in\left[0, T_{u}\right]$. If $t \in\left[\sum_{i=0}^{n} t_{i}, \sum_{i=0}^{n+1} t_{i}\right], n=0, \ldots, k-1$, then $\widehat{\varphi}_{u}(t)=\varphi\left(\left(\alpha_{1}, t_{1}\right) \ldots\left(\alpha_{n}, t-\sum_{i=0}^{n} t_{i}\right)\right)$. The derivation $\left(D_{\alpha} \varphi\right)(u)$ is well defined if the function $\widehat{\varphi}_{(u)(\alpha, t)}(\hat{t})=\varphi((u)(\alpha, \hat{t}))$, $\hat{t} \in\left[T_{u}, T_{u}+t\right]$ is differentiable at $T_{u}+$.

We say that the map $\varphi: \widetilde{\mathcal{U}_{p c}} \rightarrow \mathbb{R}$ is smooth if the derivations $D_{\alpha_{1}} \ldots D_{\alpha_{i}} \varphi$ are well defined on $\widetilde{\mathcal{U}_{p c}}$ for every $i \in \mathbb{N}$ and $\alpha_{j} \in U, j=1, \ldots, i$. To simplify the notation, the derivation $D_{\alpha_{1}} \ldots D_{\alpha_{i}} \varphi$ can be rewritten as $D_{\alpha} \varphi$, where $\alpha=\left(\alpha_{1}, \ldots, \alpha_{i}\right)$.

DeFINITION 4.3. Consider a set $\widetilde{\mathcal{U}_{p c}}$ of admissible inputs with the values in $U \subseteq \mathbb{R}^{m}$. We say that a function $\varphi: \widetilde{\mathcal{U}_{p c}} \rightarrow \mathbb{R}$ is analytic at the switching timepoints of the inputs from $\widetilde{\mathcal{U}_{p c}}$ if for every input $u=\left(u_{1}, t_{1}\right) \ldots\left(u_{k}, t_{k}\right) \in \widetilde{\mathcal{U}_{p c}}$ the function

$$
\varphi_{u_{1}, \ldots, u_{k}}\left(t_{1}, \ldots, t_{k}\right)=\varphi\left(\left(u_{1}, t_{1}\right) \ldots\left(u_{k}, t_{k}\right)\right)
$$

is analytic, i.e., we can write $\varphi_{u_{1}, \ldots, u_{k}}$ in the form of convergent formal power series in $k$ indeterminates. We denote the set of real functions $\varphi: \widetilde{\mathcal{U}_{p c}} \rightarrow \mathbb{R}$ which are analytic at the switching time-points of the inputs from $\widetilde{\mathcal{U}_{p c}}$ by $\mathcal{A}\left(\widetilde{\mathcal{U}_{p c}} \rightarrow \mathbb{R}\right)$. We refer to the elements of $\mathcal{A}\left(\widetilde{\mathcal{U}_{p c}} \rightarrow \mathbb{R}\right)$ as to the analytic functions on $\widetilde{\mathcal{U}_{p c}}$.

Let $\varphi$ be an analytic function on $\widetilde{\mathcal{U}_{p c}}$. Then for every $(u)(\alpha, 0)(v) \in \widetilde{\mathcal{U}_{p c}}$

$$
\varphi((u)(\alpha, 0)(v))=\varphi((u)(v)) .
$$

THEOREM 4.4. The set $\mathcal{A}\left(\widetilde{\mathcal{U}_{p c}} \rightarrow \mathbb{R}\right)$ of analytic functions on a set $\widetilde{\mathcal{U}_{p c}}$ of admissible inputs with the values in $U \subseteq \mathbb{R}^{m}$ is an integral domain.

Proof. To prove that $\mathcal{A}\left(\widetilde{\mathcal{U}_{p c}} \rightarrow \mathbb{R}\right)$ is an integral domain, we prove that for $f, g \in \mathcal{A}\left(\widetilde{\mathcal{U}_{p c}} \rightarrow \mathbb{R}\right)$ it holds that if $f g=0$ on $\widetilde{\mathcal{U}_{p c}}$, then $f=0$ on $\widetilde{\mathcal{U}_{p c}}$ or $g=0$ on $\widetilde{\mathcal{U}_{p c}}$.

Consider $f, g \in \mathcal{A}\left(\widetilde{\mathcal{U}_{p c}} \rightarrow \mathbb{R}\right)$ such that $f g=0$. Then $f g(u)=0$ for every $u \in \widetilde{\mathcal{U}_{p c}}$. Let $u \in \widetilde{\mathcal{U}_{p c}}$ be an arbitrary input. Because $f(u), g(u) \in \mathbb{R}$ and because $\mathbb{R}$ is an integral domain, the equality $f g(u)=f(u) g(u)=0$ implies that either $f(u)=0$ or $g(u)=0$.

To complete the proof we have to prove that it is not possible that there exist $u, v \in \widetilde{\mathcal{U}_{p c}}$ such that $f(u)=g(v)=0$ and $f(v), g(u) \neq 0$. Let us assume for a contradiction that there exist such $u=\left(\alpha_{1}, t_{1}^{u}\right) \ldots\left(\alpha_{k}, t_{k}^{u}\right), v=\left(\beta_{1}, t_{1}^{v}\right) \ldots\left(\beta_{l}, t_{l}^{v}\right) \in$ $\widetilde{\mathcal{U}_{p c}}$. Since $u, v \in \widetilde{\mathcal{U}_{p c}}$, we derive from Definition 4.1(ii) that

$$
\exists t_{i}^{v^{\prime}} \in\left[0, t_{i}^{v}\right], i=1, \ldots, l: w=\left(\alpha_{1}, t_{1}^{u}\right) \ldots\left(\alpha_{k}, t_{k}^{u}\right)\left(\beta_{1}, t_{1}^{v^{\prime}}\right) \ldots\left(\beta_{l}, t_{l}^{v^{\prime}}\right) \in \widetilde{\mathcal{U}_{p c}} .
$$

From Definition 4.1(iii), there exists $\delta>0$ such that $w^{\prime}=\left(\alpha_{1}, t_{1}^{u^{\prime}}\right) \ldots\left(\alpha_{k}, t_{k}^{u^{\prime}}\right)\left(\beta_{1}, t_{1}^{v^{\prime \prime}}\right)$ $\ldots\left(\beta_{l}, t_{l}^{v^{\prime \prime}}\right) \in \widetilde{\mathcal{U}_{p c}}$ for every $t_{i}^{u^{\prime}} \in\left[0, t_{i}^{u}+\delta\right), i=1, \ldots, k$, and for every $t_{j}^{v^{\prime \prime}} \in\left[0, t_{j}^{v^{\prime}}+\right.$ $\delta), j=1, \ldots, l$. Because $f g=0$ on $\widetilde{\mathcal{U}_{p c}}$, it follows that $0=f\left(w^{\prime}\right) g\left(w^{\prime}\right)$, and thus $0=$ $f_{\alpha_{1}, \ldots, \alpha_{k}, \beta_{1}, \ldots, \beta_{l}}\left(t_{1}^{u^{\prime}}, \ldots, t_{k}^{u^{\prime}}, t_{1}^{v^{\prime \prime}}, \ldots, t_{k}^{v^{\prime \prime}}\right) g_{\alpha_{1}, \ldots, \alpha_{k}, \beta_{1}, \ldots, \beta_{l}}\left(t_{1}^{u^{\prime}}, \ldots, t_{k}^{u^{\prime}}, t_{1}^{v^{\prime \prime}}, \ldots, t_{k}^{v^{\prime \prime}}\right)$ for $t_{i}^{u^{\prime}}$ $\in\left[0, t_{i}^{u}+\delta\right), t_{j}^{v^{\prime \prime}} \in\left[0, t_{j}^{v^{\prime}}+\delta\right), i=1, \ldots, k, j=1, \ldots, l$. Hence,

$$
f_{\alpha_{1}, \ldots, \alpha_{k}, \beta_{1}, \ldots, \beta_{l}} g_{\alpha_{1}, \ldots, \alpha_{k}, \beta_{1}, \ldots, \beta_{l}}=0 \text { on } \prod_{i=1, \ldots, k}\left[0, t_{i}^{u}+\delta\right) \times \prod_{j=1, \ldots, l}\left[0, t_{i}^{v^{\prime}}+\delta\right) .
$$

Because $f, g \in \mathcal{A}\left(\widetilde{\mathcal{U}_{p c}} \rightarrow \mathbb{R}\right)$, it holds that $f_{\alpha_{1}, \ldots, \alpha_{k}, \beta_{1}, \ldots, \beta_{l}}$ and $g_{\alpha_{1}, \ldots, \alpha_{k}, \beta_{1}, \ldots, \beta_{l}}$ are convergent formal power series in $k+l$ indeterminates for all $\alpha_{1}, \ldots, \alpha_{k}, \beta_{1}, \ldots, \beta_{l} \in U$,

Copyright (c) by SIAM. Unauthorized reproduction of this article is prohibited. 
and $k, l \in \mathbb{N}$. Since $\prod_{i=1, \ldots, k}\left[0, t_{i}^{u}+\delta\right) \times \prod_{j=1, \ldots, l}\left[0, t_{i}^{v^{\prime}}+\delta\right)$ is an open connected set in $\mathbb{R}^{k+l}$, from (4.2) and from the fact that a ring of convergent formal power series over $\mathbb{R}$ in finitely many indeterminates is an integral domain (this follows from $[20$, Volume 2, Chapter 7, Theorem 1]), we derive that either

$$
f_{\alpha_{1}, \ldots, \alpha_{k}, \beta_{1}, \ldots, \beta_{l}}=0 \text { on } \prod_{i=1, \ldots, k}\left[0, t_{i}^{u}+\delta\right) \times \prod_{j=1, \ldots, l}\left[0, t_{j}^{v^{\prime}}+\delta\right)
$$

or

$$
g_{\alpha_{1}, \ldots, \alpha_{k}, \beta_{1}, \ldots, \beta_{l}}=0 \text { on } \prod_{i=1, \ldots, k}\left[0, t_{i}^{u}+\delta\right) \times \prod_{j=1, \ldots, l}\left[0, t_{j}^{v^{\prime}}+\delta\right) .
$$

By assuming that either (4.3) or (4.4) holds, we come to a contradiction. This completes the proof.

Let us assume that (4.3) holds. Therefore, for $\tau_{i} \in\left[0, t_{i}^{u}+\delta\right), \tau_{j}^{\prime} \in\left[0, t_{j}^{v^{\prime}}+\delta\right), i=$ $1, \ldots, k, j=1, \ldots, l$, we get that $f_{\alpha_{1}, \ldots, \alpha_{k}, \beta_{1}, \ldots, \beta_{l}}\left(\tau_{1}, \ldots, \tau_{k}, \tau_{1}^{\prime}, \ldots, \tau_{l}^{\prime}\right)=0$. Because $f_{\alpha_{1}, \ldots, \alpha_{k}, \beta_{1}, \ldots, \beta_{l}}\left(0, \ldots, 0, \tau_{1}^{\prime}, \ldots, \tau_{l}^{\prime}\right)=f_{\beta_{1}, \ldots, \beta_{l}}\left(\tau_{1}^{\prime}, \ldots, \tau_{l}^{\prime}\right)$, we derive that $f_{\beta_{1}, \ldots, \beta_{l}}\left(\tau_{1}^{\prime}\right.$, $\left.\ldots, \tau_{l}^{\prime}\right)=0$ for $\tau_{j}^{\prime} \in\left[0, t_{j}^{v^{\prime}}+\delta\right), j=1, \ldots, l$. Further, because $v \in \widetilde{\mathcal{U}_{p c}}$, from Definition 4.1(iii) it follows that

$$
\exists \epsilon>0 \forall t_{j} \in\left[0, t_{j}^{v}+\epsilon\right), j=1, \ldots, l:\left(\beta_{1}, t_{1}\right) \ldots\left(\beta_{l}, t_{l}\right) \in \widetilde{\mathcal{U}_{p c}} .
$$

Thus, $f_{\beta_{1}, \ldots, \beta_{l}}$ can be represented as a convergent formal power series in $l$ indeterminates with a convergence domain containing $\prod_{j=1, \ldots, l}\left[0, t_{j}^{v}+\epsilon\right)$. Since $f_{\beta_{1}, \ldots, \beta_{l}}=0$ on $\prod_{j=1, \ldots, l}\left[0, t_{j}^{v^{\prime}}+\delta\right)$, then $f_{\beta_{1}, \ldots, \beta_{l}}=0$ also on $\prod_{j=1, \ldots, l}\left[0, t_{j}^{v}+\epsilon\right)$. Therefore, $f_{\beta_{1}, \ldots, \beta_{l}}\left(t_{1}^{v}, \ldots, t_{l}^{v}\right)=f(v)=0$, which contradicts the assumption $f(v) \neq 0$.

Let us assume that (4.4) holds. Then $g_{\alpha_{1}, \ldots, \alpha_{k}, \beta_{1}, \ldots, \beta_{l}}\left(\tau_{1}, \ldots, \tau_{k}, \tau_{1}^{\prime}, \ldots, \tau_{l}^{\prime}\right)=$ $0 \forall \tau_{i} \in\left[0, t_{i}^{u}+\delta\right), \tau_{j}^{\prime} \in\left[0, t_{j}^{v^{\prime}}+\delta\right), i=1, \ldots, k, j=1, \ldots, l$, and thus especially for $\tau_{i}=t_{i}^{u}, i=1, \ldots, k$, and $\tau_{j}^{\prime}=0, j=1, \ldots, l$. Therefore,

$$
0=g_{\alpha_{1}, \ldots, \alpha_{k}, \beta_{1}, \ldots, \beta_{l}}\left(t_{1}, \ldots, t_{k}, 0, \ldots, 0\right)=g_{\alpha_{1}, \ldots, \alpha_{k}}\left(t_{1}, \ldots, t_{k}\right)=g(u),
$$

which contradicts the assumption $g(u) \neq 0$.

Corollary 4.5. Because the set $\mathcal{A}\left(\widetilde{\mathcal{U}_{p c}} \rightarrow \mathbb{R}\right)$ is an integral domain, we can define the field $\mathcal{Q}\left(\widetilde{\mathcal{U}_{p c}} \rightarrow \mathbb{R}\right)$ of the quotients of elements of $\mathcal{A}\left(\widetilde{\mathcal{U}_{p c}} \rightarrow \mathbb{R}\right)$.

\section{Rational realizations.}

Response maps. In this paper we work with response maps rather than with input/output (I/O) maps, since it is technically more convenient. The I/O maps are considered to be the maps between the spaces of input and output functions (functions of time) mapping an input to an output. We call a map which describes the outputs immediately after applying finite parts of the inputs a response map.

Definition 5.1. Let $\widetilde{\mathcal{U}_{p c}}$ be a set of admissible inputs. A map $p: \widetilde{\mathcal{U}_{p c}} \rightarrow \mathbb{R}^{r}$ is called a response map if its components $p_{i}: \widetilde{\mathcal{U}_{p c}} \rightarrow \mathbb{R}, i=1, \ldots, r$, are such that $p_{i} \in \mathcal{A}\left(\widetilde{\mathcal{U}_{p c}} \rightarrow \mathbb{R}\right)$.

We posted extra assumptions on response maps in the definition above because to solve the problem of realization of a response map by a rational system, we use 
the objects like observation algebra and observation field of a response map; see Definition 5.9. These assumptions are necessary for well definedness of these objects.

Problem formulation. A rational system which for each input gives us the same output as a response map $p$ is called a rational realization of $p$ (a rational system realizing $p$ ). The realization problem for rational systems can be understood as the problem of finding such a rational system for a given map. Formally we state the problem of realization of a response map by a rational system as follows: Let $\widetilde{\mathcal{U}_{p c}}$ be a set of admissible inputs. Consider a response map $p: \widetilde{\mathcal{U}_{p c}} \rightarrow \mathbb{R}^{r}$. The realization problem for rational systems consists of determining a rational system $\Sigma=\left(X, f, h, x_{0}\right)$ such that

$$
p(u)=h\left(x\left(T_{u} ; x_{0}, u\right)\right) \forall u \in \widetilde{\mathcal{U}_{p c}} \text { and } \widetilde{\mathcal{U}_{p c}} \subseteq \mathcal{U}_{p c}(\Sigma) .
$$

Properties of rational realizations. Note that the solution of a rational system $\Sigma=\left(X, f, h, x_{0}\right)$ corresponding to an input $u \in \widetilde{\mathcal{U}_{p c}}$ is a piecewise-analytic map such that the intervals on which the solution is analytic are determined by the switching time-points of the input $u$.

DEFINITION 5.2. Let $\Sigma=\left(X, f, h, x_{0}\right)$ be a rational realization of a response map $p: \widetilde{\mathcal{U}_{p c}} \rightarrow \mathbb{R}^{r}$, where $\widetilde{\mathcal{U}_{p c}}$ is a set of admissible inputs. Let $A$ denote the algebra of polynomial functions on $X$. We define the input-to-state map $\tau: \widetilde{\mathcal{U}_{p c}} \rightarrow X$ as the map $\tau(u)=x\left(T_{u} ; x_{0}, u\right)$ for $u \in \widetilde{\mathcal{U}_{p c}}$. The map $\tau^{*}$ determined by $\tau$ is defined as $\tau^{*}: A \rightarrow \mathcal{A}\left(\widetilde{\mathcal{U}_{p c}} \rightarrow \mathbb{R}\right)$ such that $\tau^{*}(\varphi)=\varphi \circ \tau \forall \varphi \in A$.

To simplify further reference, we state some properties of the map $\tau^{*}$ in Proposition 5.3. The proof of this proposition is omitted because it directly follows from the definition of $\tau^{*}$.

Proposition 5.3. Let $\Sigma=\left(X, f, h, x_{0}\right)$ be a rational realization of a response map $p: \widetilde{\mathcal{U}_{p c}} \rightarrow \mathbb{R}^{r}$, where $\widetilde{\mathcal{U}_{p c}}$ is a set of admissible inputs. Let $A$ be the algebra of polynomials on $X$, let $\varphi_{1}, \ldots, \varphi_{k} \in A, k<\infty$ be such that $A=\mathbb{R}\left[\varphi_{1}, \ldots, \varphi_{k}\right]$, and let $Q$ denote the field of rational functions on $X$. Then the map $\tau^{*}: A \rightarrow$ $\mathcal{A}\left(\widetilde{\mathcal{U}_{p c}} \rightarrow \mathbb{R}\right)$ defined in Definition 5.2 is a homomorphism, and $\tau^{*}\left(\mathbb{R}\left[\varphi_{1}, \ldots, \varphi_{k}\right]\right)=$ $\mathbb{R}\left[\tau^{*} \varphi_{1}, \ldots, \tau^{*} \varphi_{k}\right]$. Moreover, the map $\widehat{\tau^{*}}: A / \operatorname{Ker} \tau^{*} \rightarrow \mathbb{R}\left[\tau^{*} \varphi_{1}, \ldots, \tau^{*} \varphi_{k}\right]$, defined as $\widehat{\tau^{*}}([\varphi])=\tau^{*} \varphi$ for every $\varphi \in A$, is an isomorphism. The map $\widehat{\tau^{*}}$ can be extended to an isomorphism of the fields $\mathcal{Q}\left(A / \operatorname{Ker} \tau^{*}\right)$ and $\mathbb{R}\left(\tau^{*} \varphi_{1}, \ldots, \tau^{*} \varphi_{k}\right)$.

The definitions of algebraic reachability (Definition 5.4) and rational observability (Definition 5.6) of rational realizations are based on [3, Definition 3,4].

DEFINITION 5.4. Let $\Sigma=\left(X, f, h, x_{0}\right)$ be a rational realization of a response map $p: \widetilde{\mathcal{U}_{p c}} \rightarrow \mathbb{R}^{r}$, where $\widetilde{\mathcal{U}_{p c}}$ is a set of admissible inputs. The rational system $\Sigma$ is said to be algebraically reachable (from the initial state) $x_{0}$ if the reachable set

$$
\mathcal{R}\left(x_{0}\right)=\left\{x\left(T_{u} ; x_{0}, u\right) \in X \mid u \in \widetilde{\mathcal{U}_{p c}} \subseteq \mathcal{U}_{p c}(\Sigma)\right\}
$$

is Z-dense in $X$.

Proposition 5.5. Let $\Sigma=\left(X, f, h, x_{0}\right)$ be a rational system as in Definition 5.4. Then the closure $Z-\operatorname{cl}\left(\mathcal{R}\left(x_{0}\right)\right)$ of the reachable set $\mathcal{R}\left(x_{0}\right)$ in Zariski topology on $X$ is an irreducible variety.

Proof. The Zariski closure of the reachable set $\mathcal{R}\left(x_{0}\right)$ is the smallest variety in $X$ containing $\mathcal{R}\left(x_{0}\right)$, which is given as $Z$-cl $\left(\mathcal{R}\left(x_{0}\right)\right)=\{x \in X \mid \varphi(x)=0 \forall \varphi \in$ $A$ such that $\varphi=0$ on $\left.\mathcal{R}\left(x_{0}\right)\right\}$. Therefore, by considering $\tau: \widetilde{\mathcal{U}_{p c}} \rightarrow X$ and $\tau^{*}: A \rightarrow$ 
$\mathcal{A}\left(\widetilde{\mathcal{U}_{p c}} \rightarrow \mathbb{R}\right)$ defined in Definition 5.2, we derive that

$$
\begin{aligned}
Z-\operatorname{cl}\left(\mathcal{R}\left(x_{0}\right)\right) & =\left\{x \in X \mid \varphi(x)=0 \forall \varphi \in A \text { such that } \varphi \circ \tau=0 \text { on } \widetilde{\mathcal{U}_{p c}}\right\} \\
& =\left\{x \in X \mid \varphi(x)=0 \forall \varphi \in A \text { such that } \tau^{*} \varphi=0\right\} \\
& =\left\{x \in X \mid \varphi(x)=0 \forall \varphi \in \operatorname{Ker} \tau^{*}\right\} .
\end{aligned}
$$

Because $\tau^{*}$ is a homomorphism and thus $\tau^{*}(A)$ is a subalgebra of $\mathcal{A}\left(\widetilde{\mathcal{U}_{p c}} \rightarrow \mathbb{R}\right)$ and because $\mathcal{A}\left(\widetilde{\mathcal{U}_{p c}} \rightarrow \mathbb{R}\right)$ is an integral domain, $\tau^{*}(A)$ is an integral domain. From Proposition 5.3, the map $\widehat{\tau^{*}}: A / \operatorname{Ker} \tau^{*} \rightarrow \tau^{*}(A)$ is an isomorphism. This implies, since $\tau^{*}(A)$ is an integral domain, that $\operatorname{Ker} \tau^{*}$ is a prime ideal.

According to [7, Chapter 5.1, Proposition 4], an affine variety $V$ is irreducible if and only if the ideal of polynomials which vanish on $V$ is a prime ideal. Because, the variety $Z-\operatorname{cl}\left(\mathcal{R}\left(x_{0}\right)\right)$ is determined by the polynomial ideal $\operatorname{Ker} \tau^{*}$ and because $\operatorname{Ker} \tau^{*}$ is a prime ideal, the variety $Z-\operatorname{cl}\left(\mathcal{R}\left(x_{0}\right)\right)$ is irreducible.

Definition 5.6. Let $\Sigma=\left(X, f=\left\{f_{\alpha} \mid \alpha \in U\right\}, h, x_{0}\right)$ be a rational system, and let $Q$ denote the field of rational functions on $X$. The observation algebra $A_{\text {obs }}(\Sigma)$ of $\Sigma$ is the smallest subalgebra of the field $Q$ containing all components $h_{i}, i=1, \ldots, r$, of $h$ and closed with respect to the derivations given by rational vector fields $f_{\alpha}, \alpha \in U$. The observation field $Q_{o b s}(\Sigma)$ of the system $\Sigma$ is the field of quotients of $A_{o b s}(\Sigma)$. The rational system $\Sigma$ is called rationally observable if $Q_{\text {obs }}(\Sigma)=Q$.

The observation algebra of a rational system is an integral domain because it is a subalgebra of an integral domain. Therefore, the observation field of a rational system is well defined. The observation field $Q_{o b s}(\Sigma)$ is also closed with respect to the derivations given by rational vector fields $f_{\alpha}, \alpha \in U$.

Proposition 5.7 (see [3], Proposition 1). For a rational system $\Sigma, Q_{o b s}(\Sigma)$ is a finitely generated field extension of $\mathbb{R}$, i.e., there exist $\varphi_{1}, \ldots, \varphi_{k} \in Q_{o b s}(\Sigma)$ such that $Q_{\text {obs }}(\Sigma)=\mathbb{R}\left(\varphi_{1}, \ldots, \varphi_{k}\right)$.

DEFINITION 5.8. We call a rational realization of a response map canonical if it is both rationally observable and algebraically reachable.

In realization theory one is given a response map and is supposed to find a system within a certain class of systems which corresponds to this map. This map is therefore representing the unknown system. For this reason, it is also useful to define objects as observation algebra and observation field for response maps.

Definition 5.9. Let $\widetilde{\mathcal{U}_{p c}}$ be a set of admissible inputs, and let $p: \widetilde{\mathcal{U}_{p c}} \rightarrow \mathbb{R}^{r}$ be a response map. The observation algebra $A_{\text {obs }}(p)$ of $p$ is the smallest subalgebra of the algebra $\mathcal{A}\left(\widetilde{\mathcal{U}_{p c}} \rightarrow \mathbb{R}\right)$ which contains the components $p_{i}, i=1, \ldots, r$ of $p$, and which is closed with respect to the derivations $D_{\alpha}, \alpha \in U$. The observation field $Q_{o b s}(p)$ of $p$ is the field of quotients of $A_{\text {obs }}(p)$.

Note that the observation field $Q_{o b s}(p)$ of $p$ is well defined only if $A_{o b s}(p)$ is an integral domain. This is the case for response maps because the components of a response map are the elements of $\mathcal{A}\left(\widetilde{\mathcal{U}_{p c}} \rightarrow \mathbb{R}\right)$ for a set $\widetilde{\mathcal{U}_{p c}}$ of admissible inputs and because $\mathcal{A}\left(\widetilde{\mathcal{U}_{p c}} \rightarrow \mathbb{R}\right)$, and thus also $\mathcal{Q}\left(\widetilde{\mathcal{U}_{p c}} \rightarrow \mathbb{R}\right)$ is an integral domain. For well definedness of the observation algebra of $p$, it is sufficient to assume that the components of $p$ are smooth (with respect to $D_{\alpha}$ derivations).

Existence of rational realizations. We provide sufficient and necessary conditions for a response map to be realizable by a rational system. The realizability of response maps by a polynomial system is treated in [4, Theorem 2]. The proof of that theorem and the proof of Proposition 5.14 have the same structure. The following lemma can be found in [4] where it is stated for polynomial systems. 
LEMMA 5.10. Let $\Sigma=\left(X, f, h, x_{0}\right)$ be a rational realization of a response map $p: \widetilde{\mathcal{U}_{p c}} \rightarrow \mathbb{R}^{r}$, where $\widetilde{\mathcal{U}_{p c}}$ is a set of admissible inputs, and let $\tau: \widetilde{\mathcal{U}_{p c}} \rightarrow X$ be as in Definition 5.2. Then for any $\varphi$ from the algebra $A$ of polynomials on $X$ and for any $\alpha \in U$ where $U$ is the set of values of the inputs of $\mathcal{U}_{p c}$, it holds that $D_{\alpha}(\varphi \circ \tau)=$ $\left(f_{\alpha} \varphi\right) \circ \tau$.

Proof. Let $u \in \widetilde{\mathcal{U}_{p c}}$, and let $\alpha \in U$. Because $\widetilde{\mathcal{U}_{p c}} \subseteq \mathcal{U}_{p c}(\Sigma)$, the trajectories of the rational system $\Sigma$ corresponding to the input $(u)(\alpha, s)$ with sufficiently small $s>0$ and to all restrictions of $(u)(\alpha, s)$ to shorter time domains are well defined. From Definition 4.2 we get that for arbitrary $\varphi \in A$

$$
D_{\alpha}(\varphi \circ \tau)(u)=\left.\frac{d}{d s}(\varphi \circ \tau)((u)(\alpha, s))\right|_{s=0+}=\left.\frac{d}{d s} \varphi(\tau((u)(\alpha, s)))\right|_{s=0+} .
$$

By the definition of an input-to-state map $\tau, \tau((u)(\alpha, s))=x\left(T_{u}+s ; x_{0},(u)(\alpha, s)\right)$. Further, according to Definition 2.2,

$$
D_{\alpha}(\varphi \circ \tau)(u)=\left.\left(f_{\alpha} \varphi\right)\left(x\left(T_{u}+s ; x_{0},(u)(\alpha, s)\right)\right)\right|_{s=0+} .
$$

Finally, by the continuity of rational function $f_{\alpha} \varphi$ along the trajectory of $\Sigma$ determined by the input $(u)(\alpha, s)$ and by the properties of a trajectory, we get that $D_{\alpha}(\varphi \circ \tau)(u)=$ $\left(f_{\alpha} \varphi\right)(\tau(u))$.

Proposition 5.11. Let $p: \widetilde{\mathcal{U}_{p c}} \rightarrow \mathbb{R}^{r}$ be a response map realizable by a rational system $\Sigma=\left(X, f, h, x_{0}\right)$. Let $\tau: \widetilde{\mathcal{U}_{p c}} \rightarrow X$ be as in Definition 5.2. Then the map $\tau_{\text {ext }}^{*}: A_{\text {obs }}(\Sigma) \rightarrow A_{\text {obs }}(p)$ defined as $\tau_{\text {ext }}^{*} \varphi=\varphi \circ \tau$ for every $\varphi \in \mathcal{A}_{\text {obs }}(\Sigma)$ is a welldefined surjective homomorphism, i.e., $\tau_{\text {ext }}^{*}\left(A_{o b s}(\Sigma)\right)=A_{\text {obs }}(p)$.

Proof. Note that $\tau_{e x t}^{*}$ is defined in the same way as $\tau^{*}$ but on a different domain; see Definition 5.2. Obviously, $\tau_{e x t}^{*}$ is a homomorphism. We prove that $\tau_{e x t}^{*}$ is well defined and that it is surjective.

By Definitions 5.6 and 5.9, the observation algebras of a system $\Sigma$ and of a map $p$ are generated by $h_{i}, f_{\alpha_{1}} \ldots f_{\alpha_{j}} h_{i}$ and $p_{i}, D_{\alpha_{1}} \ldots D_{\alpha_{j}} p_{i}$, respectively, such that $j \in \mathbb{N}$, $\alpha_{1}, \ldots, \alpha_{j} \in U$, and $i=1, \ldots, r$. Since $\tau_{e x t}^{*}$ is a homomorphism, we prove that $\tau_{e x t}^{*}\left(A_{o b s}(\Sigma)\right)=A_{o b s}(p)$ by proving that the generators of $A_{o b s}(\Sigma)$ and $A_{o b s}(p)$ are mapped to each other by $\tau_{e x t}^{*}$. To prove that $\tau_{e x t}^{*}$ is well defined, it is sufficient to prove that $\tau_{e x t}^{*}$ is well defined for the generators of the algebra $A_{o b s}(\Sigma)$.

Since $\Sigma$ is a rational realization of $p$, we know that $p=h \circ \tau$ and that $p$ is well defined. Because $h \in A_{\text {obs }}(\Sigma), p=\tau_{e x t}^{*} h$ which implies that $\tau_{\text {ext }}^{*}$ is well defined at $h$. Let $h_{\text {num }}, h_{\text {den }} \in A$ be such that $h=\frac{h_{n u m}}{h_{d e n}}$. For a rational vector field $f_{\alpha} \in f$, it holds that

$$
\left(f_{\alpha} h\right) \circ \tau=\left(f_{\alpha} \frac{h_{n u m}}{h_{d e n}}\right) \circ \tau=\frac{\left(f_{\alpha} h_{n u m} \circ \tau\right)\left(h_{d e n} \circ \tau\right)-\left(f_{\alpha} h_{d e n} \circ \tau\right)\left(h_{n u m} \circ \tau\right)}{\left(h_{d e n} \circ \tau\right)^{2}},
$$

and further, by Lemma 5.10, that

$$
\left(f_{\alpha} h\right) \circ \tau=\frac{D_{\alpha}\left(h_{n u m} \circ \tau\right)\left(h_{\text {den }} \circ \tau\right)-D_{\alpha}\left(h_{\text {den }} \circ \tau\right)\left(h_{n u m} \circ \tau\right)}{\left(h_{\text {den }} \circ \tau\right)^{2}} .
$$

Therefore, $\tau_{\text {ext }}^{*}\left(f_{\alpha} h\right)=\left(f_{\alpha} h\right) \circ \tau=D_{\alpha}\left(\frac{h_{n u m}}{h_{\text {den }}} \circ \tau\right)=D_{\alpha}(h \circ \tau)=D_{\alpha}(p)$. As $p$ is a response map, the derivations $D_{\alpha}$ of $p$ are well defined, and consequently $\tau_{e x t}^{*}$ is well defined at $f_{\alpha} h \in A_{o b s}(\Sigma) \subseteq Q$.

In the next proposition we state necessary conditions for a response map to be realizable by a rational system. 
Proposition 5.12. Let $p: \widetilde{\mathcal{U}_{p c}} \rightarrow \mathbb{R}^{r}$ be a response map realizable by a rational system $\Sigma=\left(X, f, h, x_{0}\right)$. Let $\tau_{\text {ext }}^{*}: A_{\text {obs }}(\Sigma) \rightarrow A_{\text {obs }}(p)$ be as in Proposition 5.11. Then

(i) $Q_{o b s}(p)=\widehat{\tau^{*}}\left(\mathcal{Q}\left(A_{o b s}(\Sigma) / \operatorname{Ker} \tau_{\text {ext }}^{*}\right)\right)$, where $\widehat{\tau^{*}}: A_{\text {obs }}(\Sigma) / \operatorname{Ker} \tau_{\text {ext }}^{*} \rightarrow A_{o b s}(p)$ is an isomorphism derived from the map $\tau_{\text {ext }}^{*}: A_{\text {obs }}(\Sigma) \rightarrow A_{\text {obs }}(p)$;

(ii) $Q_{o b s}(p)$ is finitely generated.

Proof. (i) According to Proposition 5.11, the map $\tau_{\text {ext }}^{*}: A_{o b s}(\Sigma) \rightarrow A_{o b s}(p)$ is a surjective homomorphism which is not necessarily injective. Then the map

$$
\widehat{\tau^{*}}: A_{o b s}(\Sigma) / \operatorname{Ker} \tau_{e x t}^{*} \rightarrow A_{o b s}(p)
$$

defined as $\widehat{\tau^{*}}([\varphi])=\tau_{e x t}^{*}(\varphi)$ for every $\varphi \in A_{o b s}(\Sigma)$, is an isomorphism. Since the algebras $A_{o b s}(\Sigma) / \operatorname{Ker} \tau_{e x t}^{*}$ and $A_{o b s}(p)$ are integral domains, we can construct the fields of fractions of $A_{o b s}(\Sigma) / \operatorname{Ker} \tau_{e x t}^{*}$ and $A_{o b s}(p)$. We extend the isomorphism $\widehat{\tau^{*}}$ of the algebras $A_{o b s}(\Sigma) / \operatorname{Ker} \tau_{\text {ext }}^{*}$ and $A_{o b s}(p)$ to the isomorphism $\widehat{\tau^{*}}$ of the fields $\mathcal{Q}\left(A_{o b s}(\Sigma) / \operatorname{Ker} \tau_{\text {ext }}^{*}\right)$ and $Q_{o b s}(p)$. Then $Q_{o b s}(p)=\widehat{\tau^{*}}\left(\mathcal{Q}\left(A_{o b s}(\Sigma) / \operatorname{Ker} \tau_{\text {ext }}^{*}\right)\right)$.

(ii) The field $\mathcal{Q}\left(A_{o b s}(\Sigma) / \operatorname{Ker} \tau_{\text {ext }}^{*}\right)$ is a field isomorphic to a subfield $F$ of $Q_{o b s}(\Sigma)$. From [5, Chapter V, section 14.7, Corollary 3], it follows that if $G$ is a finitely generated field containing $\mathbb{R}$, then every subfield $F$ of $G$ containing $\mathbb{R}$ is finitely generated. Then, since $F \subseteq Q_{o b s}(\Sigma) \subseteq Q$ and $Q$ is a finite field extension of $\mathbb{R}$, it follows that $F$ is also finitely generated. Because a field isomorphic to a finitely generated field is finitely generated, there exist $\varphi_{1}, \ldots, \varphi_{k} \in \mathcal{Q}\left(A_{o b s}(\Sigma) / \operatorname{Ker} \tau_{e x t}^{*}\right)$ such that

$$
\mathcal{Q}\left(A_{o b s}(\Sigma) / \operatorname{Ker} \tau_{e x t}^{*}\right)=\mathbb{R}\left(\varphi_{1}, \ldots, \varphi_{k}\right) \text {. }
$$

From (i) of Proposition 5.12, $Q_{o b s}(p)=\widehat{\tau^{*}}\left(\mathcal{Q}\left(A_{o b s}(\Sigma) / \operatorname{Ker} \tau_{\text {ext }}^{*}\right)\right)$, where $\widehat{\tau^{*}}$ is an isomorphism. Then, by (5.1), $Q_{o b s}(p)=\widehat{\tau^{*}}\left(\mathbb{R}\left(\varphi_{1}, \ldots, \varphi_{k}\right)\right)=\mathbb{R}\left(\widehat{\tau^{*}} \varphi_{1}, \ldots, \widehat{\tau^{*}} \varphi_{k}\right)$. Thus $Q_{o b s}(p)$ is finitely generated.

In the following proposition we prove that the generators of the observation field $Q_{o b s}(p)$ of a response map $p$ can be chosen from $\mathcal{A}\left(\widetilde{\mathcal{U}_{p c}} \rightarrow \mathbb{R}\right)$. This allows us to reformulate the necessary condition for a response map $p$ to be realizable by a rational system which is stated as the condition (ii) in the proposition above in the following way: $Q_{o b s}(p)$ is finitely generated by the elements from $\mathcal{A}\left(\widetilde{\mathcal{U}_{p c}} \rightarrow \mathbb{R}\right)$.

Proposition 5.13. Let $p: \widetilde{\mathcal{U}_{p c}} \rightarrow \mathbb{R}^{r}$ be a response map. The observation field $Q_{o b s}(p)$ is finitely generated if and only if it is finitely generated by the elements from $\mathcal{A}\left(\widetilde{\mathcal{U}_{p c}} \rightarrow \mathbb{R}\right)$, i.e., there exist finitely many $\varphi_{1}, \ldots, \varphi_{k} \in \mathcal{A}\left(\widetilde{\mathcal{U}_{p c}} \rightarrow \mathbb{R}\right)$ such that $Q_{o b s}(p)=\mathbb{R}\left(\varphi_{1}, \ldots, \varphi_{k}\right)$.

Proof. $(\Leftarrow)$ Let $Q_{\text {obs }}(p)$ be finitely generated by the elements from $\mathcal{A}\left(\widetilde{\mathcal{U}_{p c}} \rightarrow \mathbb{R}\right)$. Then it is obviously finitely generated.

$(\Rightarrow)$ Let $Q_{o b s}(p)$ be finitely generated. There exist $\varphi_{1}, \ldots, \varphi_{k} \in Q_{o b s}(p)$ such that $Q_{o b s}(p)=\mathbb{R}\left(\varphi_{1}, \ldots, \varphi_{k}\right)$. As $\varphi_{i} \in Q_{o b s}(p), i=1, \ldots, k$, we know that $\varphi_{i}=\frac{\varphi_{i, n u m}}{\varphi_{i, \text { den }}}$ where $\varphi_{i, \text { num }}, \varphi_{i, \text { den }} \in A_{\text {obs }}(p)$ for $i=1, \ldots, k$. We define the field

$$
F=\mathbb{R}\left(\varphi_{1, \text { num }}, \varphi_{1, \text { den }}, \ldots, \varphi_{k, \text { num }}, \varphi_{k, \text { den }}\right) .
$$

Because $\varphi_{i, \text { num }}, \varphi_{i, d e n} \in A_{o b s}(p)$ for $i=1, \ldots, k$, and because $A_{o b s}(p)$ is a subalgebra of $\mathcal{A}\left(\widetilde{\mathcal{U}_{p c}} \rightarrow \mathbb{R}\right)$, it follows that

$$
\begin{gathered}
\varphi_{i, \text { num }}, \varphi_{i, \text { den }} \in \mathcal{A}\left(\widetilde{\mathcal{U}_{p c}} \rightarrow \mathbb{R}\right) \text { for } i=1, \ldots, k, \text { and } \\
\mathbb{R}\left[\varphi_{1, \text { num }}, \varphi_{1, \text { den }}, \ldots, \varphi_{k, \text { num }}, \varphi_{k, \text { den }}\right] \subseteq A_{o b s}(p) .
\end{gathered}
$$

Copyright ( $)$ by SIAM. Unauthorized reproduction of this article is prohibited. 
By (5.2), the field $F$ is generated by the elements from $\mathcal{A}\left(\widetilde{\mathcal{U}_{p c}} \rightarrow \mathbb{R}\right)$. From the definition of $F$ it is obvious that $F \supseteq Q_{o b s}(p)$, and from (5.3) we get by taking the quotients that $F \subseteq Q_{o b s}(p)$. Therefore, $Q_{o b s}(p)=F$, and thus the field $Q_{o b s}(p)$ is finitely generated by the elements from $\mathcal{A}\left(\widetilde{\mathcal{U}_{p c}} \rightarrow \mathbb{R}\right)$.

In the following proposition we specify sufficient conditions for a response map to be realizable by a rational system.

Proposition 5.14. Let $p: \widetilde{\mathcal{U}_{p c}} \rightarrow \mathbb{R}^{r}$ be a response map. If there exists a field $F \subseteq \mathcal{Q}\left(\widetilde{\mathcal{U}_{p c}} \rightarrow \mathbb{R}\right)$ such that

(i) $F$ is finitely generated by the elements from $\mathcal{A}\left(\widetilde{\mathcal{U}_{p c}} \rightarrow \mathbb{R}\right)$,

(ii) $F$ is closed with respect to $D_{\alpha}$ derivations, i.e.,

$$
\forall i \in \mathbb{N}, \forall \alpha_{j} \in U, j=1, \ldots, i: D_{\alpha_{1}} \ldots D_{\alpha_{i}} F \subseteq F
$$

(iii) $Q_{o b s}(p) \subseteq F$, then $p$ has a rational realization.

Proof. Consider a response map $p: \widetilde{\mathcal{U}_{p c}} \rightarrow \mathbb{R}^{r}$. We assume that there exists a field $F \subseteq \mathcal{Q}\left(\widetilde{\mathcal{U}_{p c}} \rightarrow \mathbb{R}\right)$ satisfying (i)-(iii) of Proposition 5.14. Let $\varphi_{1}, \ldots, \varphi_{k}: \widetilde{\mathcal{U}_{p c}} \rightarrow$ $\mathbb{R} \in \mathcal{A}\left(\widetilde{\mathcal{U}_{p c}} \rightarrow \mathbb{R}\right)$ be such that $F=\mathbb{R}\left(\varphi_{1}, \ldots, \varphi_{k}\right)$. Since $F$ is closed with respect to $D_{\alpha}$ derivations, for any $\alpha=\left(\alpha_{1}, \ldots, \alpha_{j}\right)$ such that $\alpha_{1}, \ldots, \alpha_{j} \in U, j \in \mathbb{N}$, and for every $\varphi_{i}, i=1, \ldots, k$, there exists $v_{i}^{\alpha} \in \mathbb{R}\left(X_{1}, \ldots, X_{k}\right)$ such that

$$
D_{\alpha} \varphi_{i}=v_{i}^{\alpha}\left(\varphi_{1}, \ldots, \varphi_{k}\right) .
$$

Since $Q_{o b s}(p) \subseteq F$, the components of the map $p=\left(p_{1}, \ldots, p_{r}\right)$ are elements of $F$, and thus for every $p_{j}, j=1, \ldots, r$, there exists $w_{j} \in \mathbb{R}\left(X_{1}, \ldots, X_{k}\right)$ such that

$$
p_{j}=w_{j}\left(\varphi_{1}, \ldots, \varphi_{k}\right) .
$$

We prove that a rational system $\Sigma=\left(X, f, h, x_{0}\right)$, where

$$
\begin{aligned}
& X=\mathbb{R}^{k} \\
& f_{\alpha}=\sum_{i=1}^{k} v_{i}^{\alpha} \frac{\partial}{\partial x_{i}}, \quad \alpha \in U, \\
& h_{j}\left(x_{1}, \ldots, x_{k}\right)=w_{j}\left(x_{1}, \ldots, x_{k}\right), \quad j=1 \ldots r \\
& x_{0}=\left(\varphi_{1}(e), \ldots, \varphi_{k}(e)\right), e \text { is the empty input }
\end{aligned}
$$

is a rational system realizing $p$. Let us define $\Psi(t)=\left(\varphi_{1}, \ldots, \varphi_{k}\right)\left(u_{[0, t]}\right)$ for $u \in \widetilde{\mathcal{U}_{p c}}$, $t \in\left[0, T_{u}\right]$. It is well defined because $\varphi_{i} \in \mathcal{A}\left(\widetilde{\mathcal{U}_{p c}} \rightarrow \mathbb{R}\right), i=1, \ldots, k$, are defined for every $u \in \widetilde{\mathcal{U}_{p c}}$ and because $u_{[0, t]} \in \widetilde{\mathcal{U}_{p c}}$ if $u \in \widetilde{\mathcal{U}_{p c}}$ and $t \in\left[0, T_{u}\right]$. Especially, $\Psi(0)=\left(\varphi_{1}, \ldots, \varphi_{k}\right)\left(u_{[0,0]}\right)$ is well defined because $u_{[0,0]}=e \in \widetilde{\mathcal{U}_{p c}}$ and the functions $\varphi_{1}, \ldots, \varphi_{k} \in \mathcal{A}\left(\widetilde{\mathcal{U}_{p c}} \rightarrow \mathbb{R}\right)$ are defined properly at $e$. Consider a constant input $u=\left(\alpha, T_{u}\right) \in \widetilde{\mathcal{U}_{p c}}$. Note that from Definition 4.1(i), (ii), there exists $\epsilon>0$ such that for every $t, \tau \geq 0$ such that $t+\tau \in\left[0, T_{u}+\epsilon\right]$, it holds that $u^{\prime}=(\alpha, t+\tau) \in \widetilde{\mathcal{U}_{p c}}$. If $t+\tau \leq T_{u}$, then $u^{\prime}=u_{[0, t+\tau]}$, and if $T_{u}<t+\tau$, then $u=u_{\left[0, T_{u}\right]}^{\prime}$. In both cases we refer to the corresponding inputs as to the inputs $u_{[0, t+\tau]}, t+\tau \in\left[0, T_{u}+\epsilon\right]$. Then,

$$
\begin{aligned}
\Psi(0) & =\left(\varphi_{1}, \ldots, \varphi_{k}\right)\left(u_{[0,0]}\right)=\left(\varphi_{1}(e), \ldots, \varphi_{k}(e)\right)=x_{0} \text { and } \\
\frac{d}{d t} \Psi(t) & =\left.\frac{d}{d \tau} \Psi(t+\tau)\right|_{\tau=0+}=\left.\frac{d}{d \tau}\left(\varphi_{1}\left(u_{[0, t+\tau]}\right), \ldots, \varphi_{k}\left(u_{[0, t+\tau]}\right)\right)\right|_{\tau=0+} .
\end{aligned}
$$

Copyright $\odot$ by SIAM. Unauthorized reproduction of this article is prohibited. 
Because $\left(u_{[0, t]}\right)(\alpha, \tau)=u_{[0, t+\tau]}$ and because $D_{\alpha} \varphi\left(u_{[0, t]}\right)=\left.\frac{d}{d \tau} \varphi\left(\left(u_{[0, t]}\right)(\alpha, \tau)\right)\right|_{\tau=0+}$, the derivation $\frac{d}{d t} \Psi(t)$ equals $\left(D_{\alpha} \varphi_{1}\left(u_{[0, t]}\right), \ldots, D_{\alpha} \varphi_{k}\left(u_{[0, t]}\right)\right)$. Furthermore, due to (5.4), $\frac{d}{d t} \Psi(t)=\left(v_{1}^{\alpha}\left(\varphi_{1}, \ldots, \varphi_{k}\right)\left(u_{[0, t]}\right), \ldots, v_{k}^{\alpha}\left(\varphi_{1}, \ldots, \varphi_{k}\right)\left(u_{[0, t]}\right)\right)$. Finally, as $\Psi(t)=$ $\left(\varphi_{1}, \ldots, \varphi_{k}\right)\left(u_{[0, t]}\right)$, we get that

$$
\frac{d}{d t} \Psi(t)=\left(v_{1}^{\alpha}(\Psi(t)), \ldots, v_{k}^{\alpha}(\Psi(t))\right) \text { and } \Psi(0)=x_{0} .
$$

Hence $\Psi(t)=x\left(t ; x_{0}, u_{[0, t]}\right)$ for a constant input $u \in \widetilde{\mathcal{U}_{p c}}$ and for $t \in\left[0, T_{u}\right]$. This also proves that for $u=\left(\alpha_{1}, t_{1}\right) \ldots\left(\alpha_{j}, t_{j}\right) \in \widetilde{\mathcal{U}_{p c}}$ and for $t \in\left[0, t_{1}\right]$, we have $\Psi(t)=$ $x\left(t ; x_{0}, u_{[0, t]}\right)$. If we consider $u=\left(\alpha_{1}, t_{1}\right) \ldots\left(\alpha_{j}, t_{j}\right) \in \widetilde{\mathcal{U}_{p c}}$ and if we take $t \in$ $\left[t_{1}, t_{1}+t_{2}\right]$ instead of $t \in\left[0, t_{1}\right]$, we get that $\Psi\left(t_{1}\right)=x\left(t_{1} ; x_{0}, u_{\left[0, t_{1}\right]}\right)$ and $\frac{d}{d t} \Psi(t)=$ $\left(v_{1}^{\alpha_{2}}(\Psi(t)), \ldots, v_{k}^{\alpha_{2}}(\Psi(t))\right)$ for $t \in\left[t_{1}, t_{1}+t_{2}\right]$ with the same reasoning as before. For $t \in$ $\left[t_{1}, t_{1}+t_{2}\right]$, we have that $\Psi(t)=x\left(t ; \Psi\left(t_{1}\right), u_{\left[t_{1}, t_{1}+t\right]}\right)=x\left(t ; x\left(t_{1} ; x_{0}, u_{\left[0, t_{1}\right]}\right), u_{\left[t_{1}, t_{1}+t\right]}\right)$. In the analogous way, we study the cases for $t \in\left[t_{1}+t_{2}, t_{1}+t_{2}+t_{3}\right], \ldots, t \in$ $\left[t_{1}+\cdots+t_{i-1}, t_{1}+\cdots+t_{i}\right], \ldots$ Finally, $\Psi(t)=x\left(t ; x_{0}, u_{[0, t]}\right)$ for an arbitrary $u \in \widetilde{\mathcal{U}_{p c}}$ and $t \in\left[0, T_{u}\right]$. Thus the trajectories of $\Sigma$ are described by $\Psi$.

To prove that the rational system $\Sigma$ is a realization of the response map $p$, we have to prove that $p(u)=h\left(x\left(T_{u} ; x_{0}, u\right)\right)$ for every $u \in \widetilde{\mathcal{U}_{p c}}$. Consider an arbitrary $u \in \widetilde{\mathcal{U}_{p c}}$. Due to $(5.5)$,

$$
p(u)=\left(p_{1}, \ldots, p_{r}\right)(u)=\left(w_{1}\left(\varphi_{1}, \ldots, \varphi_{k}\right), \ldots, w_{r}\left(\varphi_{1}, \ldots, \varphi_{k}\right)\right)(u) .
$$

Further, by the definitions of $h_{j}, j=1, \ldots, r$, and $\Psi$, it follows that

$$
p(u)=\left(h_{1}\left(\varphi_{1}, \ldots, \varphi_{k}\right), \ldots, h_{r}\left(\varphi_{1}, \ldots, \varphi_{k}\right)\right)(u)=\left(h_{1}\left(\Psi\left(T_{u}\right)\right), \ldots, h_{r}\left(\Psi\left(T_{u}\right)\right)\right) .
$$

Finally, since $\Psi\left(T_{u}\right)=x\left(T_{u} ; x_{0}, u\right)$ for $u \in \widetilde{\mathcal{U}_{p c}}$, we derive that $p(u)=h\left(x\left(T_{u} ; x_{0}, u\right)\right)$ for $u \in \widetilde{\mathcal{U}_{p c}}$.

Remark 5.15. Proposition 5.14 can be stated as an equivalence. The proof of the other implication is the same as the sufficiency proof of Theorem 5.16.

The main theorem of this section, solving the problem of the existence of rational realizations for response maps, is based on the three propositions above.

THEOREM 5.16 (existence of rational realizations). A response map $p: \widetilde{\mathcal{U}_{p c}} \rightarrow \mathbb{R}^{r}$ has a rational realization if and only if $Q_{o b s}(p)$ is finitely generated.

Proof. $(\Rightarrow)$ See part (ii) of Proposition 5.12 for this statement and the proof.

$(\Leftarrow)$ From Proposition 5.14, the existence of a field $F \subseteq \mathcal{Q}\left(\widetilde{\mathcal{U}_{p c}} \rightarrow \mathbb{R}\right)$ finitely generated by the elements from $\mathcal{A}\left(\widetilde{\mathcal{U}_{p c}} \rightarrow \mathbb{R}\right)$, containing $Q_{o b s}(p)$, and closed with respect to $D_{\alpha}$ derivations implies the rational realizability of $p$. Since we assume that $Q_{o b s}(p)$ is finitely generated, it follows from Proposition 5.13 and Definition 5.9 that $Q_{o b s}(p)$ has those properties. By following the steps of the proof of Proposition 5.14 for $F=Q_{o b s}(p)$, we construct a rational realization of $p$.

Example. This example is motivated by an example stated in [4]. The procedure to construct a rational system realizing a given response map is following the steps made in the proof of Proposition 5.14.

Let $\widetilde{\mathcal{U}_{p c}}$ be a set of admissible inputs with the values in $\mathbb{R}$. We determine a rational system $\Sigma$ realizing a map $p: \widetilde{\mathcal{U}_{p c}} \rightarrow \mathbb{R}$ defined as $p(u)=\exp \left(\int_{0}^{T_{u}} \frac{u(s)}{(1+s)^{2}} d s\right)$. By $u(s)$, we denote the value of an input $u \in \widetilde{\mathcal{U}_{p c}}$ at a time $s \in\left[0, T_{u}\right]$. 
First, we compute $D_{\alpha}$ derivations of $p$. Consider $\alpha_{1}, \alpha_{2} \in \mathbb{R}$, and $u \in \widetilde{\mathcal{U}_{p c}}$. For $t_{1}, t_{2}>0$ sufficiently small $(u)\left(\alpha_{1}, t_{1}\right)\left(\alpha_{2}, t_{2}\right) \in \widetilde{\mathcal{U}_{p c}}$. Then

$$
\begin{aligned}
& \left(D_{\alpha_{1}} p\right)(u)=\left.\frac{d}{d \tau} p\left((u)\left(\alpha_{1}, \tau\right)\right)\right|_{\tau=0+} \\
& =\left[\frac{d}{d \tau} \exp \left(\int_{0}^{T_{u}} \frac{u(s)}{(1+s)^{2}} d s+\int_{T_{u}}^{T_{u}+\tau} \frac{\alpha_{1}}{(1+s)^{2}} d s\right)\right]_{\tau=0+} \\
& =0+p(u) \alpha_{1}\left[\exp \left(\int_{T_{u}}^{T_{u}+\tau} \frac{\alpha_{1}}{(1+s)^{2}} d s\right)\right]_{\tau=0+}\left[\frac{d}{d \tau} \int_{T_{u}}^{T_{u}+\tau} \frac{1}{(1+s)^{2}} d s\right]_{\tau=0+} \\
& =\alpha_{1} p(u) \frac{1}{\left(1+T_{u}\right)^{2}}, \\
& \left(D_{\alpha_{2}} D_{\alpha_{1}} p\right)(u) \\
& =D_{\alpha_{2}}\left(\alpha_{1} p(u) \frac{1}{\left(1+T_{u}\right)^{2}}\right)=\alpha_{1} \frac{1}{\left(1+T_{u}\right)^{2}} D_{\alpha_{2}} p(u)+\alpha_{1} p(u) D_{\alpha_{2}} \frac{1}{\left(1+T_{u}\right)^{2}} \\
& =\frac{\alpha_{1} \alpha_{2}}{\left(1+T_{u}\right)^{4}} p(u)+\alpha_{1} p(u)\left[\frac{d}{d \tau} \frac{1}{\left(1+T_{u}+\tau\right)^{2}}\right]_{\tau=0+}=\frac{\alpha_{1} \alpha_{2} p(u)}{\left(1+T_{u}\right)^{4}}+\frac{-2 \alpha_{1} p(u)}{\left(1+T_{u}\right)^{3}} .
\end{aligned}
$$

We can compute the derivations $\left(D_{\alpha_{i}} \ldots D_{\alpha_{1}} p\right)(u)$ for any $i \in \mathbb{N}, \alpha_{j} \in \mathbb{R}, j \in 1, \ldots, i$. If we define $\varphi_{1}(u)=p(u)$ and $\varphi_{2}(u)=1+T_{u}$, then for any $i \in \mathbb{N}$ and $\alpha_{j} \in \mathbb{R}, j \in$ $1, \ldots, i$, it holds that $\left(D_{\alpha_{i}} \ldots D_{\alpha_{1}} p\right)(u) \in \mathbb{R}\left(\varphi_{1}(u), \varphi_{2}(u)\right)$. Therefore, by Definition $5.9, Q_{o b s}(p) \subseteq \mathbb{R}\left(\varphi_{1}, \varphi_{2}\right)$, and consequently $Q_{o b s}(p)$ is finitely generated. Hence, according to Theorem 5.16, there exists a rational system realizing $p$.

We construct a rational system $\Sigma=\left(X, f, h, x_{0}\right)$ realizing $p$ by following the proof of Proposition 5.14. Let us consider the field $F=\mathbb{R}\left(\varphi_{1}, \varphi_{2}\right)$. It is finitely generated, contains $Q_{o b s}(p)$, and is closed with respect to $D_{\alpha}$ derivations. The number of generators of $F$ equals 2 , which implies that the state-space $X$ can be taken as $\mathbb{R}^{2}$. To determine a family of rational vector fields $f=\left\{f_{\alpha} \mid \alpha \in \mathbb{R}\right\}$, we compute

$$
v_{1}^{\alpha}\left(\varphi_{1}, \varphi_{2}\right)=D_{\alpha} \varphi_{1}=D_{\alpha} p=\alpha \varphi_{1} \frac{1}{\varphi_{2}^{2}} \quad \text { and } \quad v_{2}^{\alpha}\left(\varphi_{1}, \varphi_{2}\right)=D_{\alpha} \varphi_{2}=1 .
$$

The last equality $D_{\alpha} \varphi_{2}=1$ holds because $D_{\alpha} \varphi_{2}(u)=\left[\frac{d}{d \tau} \varphi_{2}((u)(\alpha, \tau))\right]_{\tau=0+}=$ $\left[\frac{d}{d \tau}\left(1+T_{u}+\tau\right)\right]_{\tau=0+}=[1]_{\tau=0+}=1$ for any $u \in \widetilde{\mathcal{U}_{p c}}$. The output map $h$ is determined by a map $w$ such that $w\left(\varphi_{1}, \varphi_{2}\right)=p=\varphi_{1}$, and the initial point $x_{0}$ is given as $x_{0}=\left(\varphi_{1}(e), \varphi_{2}(e)\right)$, where $e$ is the empty input. Finally, the rational realization $\Sigma=\left(X, f, h, x_{0}\right)$ of $p$ is given as

$$
\begin{aligned}
& X=\mathbb{R}^{2}, \\
& f_{\alpha}\left(x_{1}, x_{2}\right)=v_{1}^{\alpha}\left(x_{1}, x_{2}\right) \frac{\partial}{\partial x_{1}}+v_{2}^{\alpha}\left(x_{1}, x_{2}\right) \frac{\partial}{\partial x_{2}}=\alpha \frac{x_{1}}{x_{2}^{2}} \frac{\partial}{\partial x_{1}}+\frac{\partial}{\partial x_{2}}, \quad \alpha \in \mathbb{R}, \\
& h\left(x_{1}, x_{2}\right)=w\left(x_{1}, x_{2}\right)=x_{1}, \\
& x_{0}=\left(\varphi_{1}(e), \varphi_{2}(e)\right)=(1,1) .
\end{aligned}
$$

6. Canonical rational realizations. The systems which we call canonical are called minimal by Bartosiewicz in [4]. Therefore, [4, Theorem 3] describing the conditions under which a map has a minimal polynomial realization corresponds to our Theorem 6.1 considering rational systems. The proofs are analogous. 
TheOREm 6.1. Let $p: \widetilde{\mathcal{U}_{p c}} \rightarrow \mathbb{R}^{r}$ be a response map. The following statements are equivalent:

(i) $p$ has an rationally observable rational realization,

(ii) $p$ has a canonical rational realization,

(iii) $Q_{o b s}(p)$ is finitely generated.

Proof. (i) $\Rightarrow$ (ii) Let $p: \widetilde{\mathcal{U}_{p c}} \rightarrow \mathbb{R}^{r}$ be a response map. Consider a rational system $\Sigma=\left(X, f, h, x_{0}\right)$ realizing $p$, which is rationally observable. Then $\Sigma$ is canonical if it is algebraically reachable from the initial state $x_{0}$, i.e., if $Z-\operatorname{cl}\left(\mathcal{R}\left(x_{0}\right)\right)=X$. Let us denote $X^{\prime}=Z-\operatorname{cl}\left(\mathcal{R}\left(x_{0}\right)\right)$. From Proposition 5.5, the variety $X^{\prime}$ is an irreducible real affine variety. If $X^{\prime}=X$, then the proof is complete. Let us assume that $X^{\prime} \neq X$.

Because $X^{\prime}$ is an irreducible real affine variety, we can consider $X^{\prime}$ to be a statespace of a rational system $\Sigma^{\prime}$. Let $I$ be an ideal of polynomials of $A$ which vanish on $X^{\prime}$. Then the quotient ring $A / I$ can be identified with $A^{\prime}$, the algebra of polynomials on $X^{\prime}$. We denote the corresponding bijection by $\Psi: A / I \rightarrow A^{\prime}$. This is a one-toone and onto mapping, which preserves sums and products. If we consider $\varphi^{\prime} \in A^{\prime}$ and $\varphi \in A$ such that $\Psi([\varphi])=\varphi^{\prime}$, it holds that $\varphi\left\lceil_{X^{\prime}}=\varphi^{\prime}\right.$. More details can be found in [7, Chapter 5.2]. There exist polynomials $\varphi_{1}^{\prime}, \ldots, \varphi_{k}^{\prime}$ from $A^{\prime}$ such that $A^{\prime}=\mathbb{R}\left[\varphi_{1}^{\prime}, \ldots, \varphi_{k}^{\prime}\right]$. Moreover, $A^{\prime}$ is an integral domain, and we can define the field $Q^{\prime}$ of rational functions on $X^{\prime}$ by $Q^{\prime}=\mathbb{R}\left(\varphi_{1}^{\prime}, \ldots, \varphi_{k}^{\prime}\right)$.

We define the rational system $\Sigma^{\prime}=\left(X^{\prime}, f^{\prime}, h^{\prime}, x_{0}^{\prime}\right)$ by considering the state-space $X^{\prime}$ and by deriving the family $f^{\prime}$ of rational vector fields, an output function $h^{\prime}$, and an initial state $x_{0}^{\prime}$ from the rational system $\Sigma$. As the initial state $x_{0}^{\prime}$ of $\Sigma^{\prime}$, we consider $x_{0}^{\prime}=x_{0} \in Z-\operatorname{cl}\left(\mathcal{R}\left(x_{0}\right)\right)=X^{\prime}$. The output function $h^{\prime}$ of $\Sigma^{\prime}$ is defined as

$$
\begin{aligned}
h^{\prime} & =\left(h_{1}^{\prime}, \ldots, h_{r}^{\prime}\right)=\left(\frac{h_{1, \text { num }}^{\prime}}{h_{1, \text { den }}^{\prime}}, \ldots, \frac{h_{r, \text { num }}^{\prime}}{h_{r, \text { den }}^{\prime}}\right) \\
& =\left(\frac{\Psi\left(\left[h_{1, \text { num }}\right]\right)}{\Psi\left(\left[h_{1, \text { den }}\right]\right)}, \ldots, \frac{\Psi\left(\left[h_{r, \text { num }}\right]\right)}{\Psi\left(\left[h_{r, \text { den }}\right]\right)}\right)=\left(\frac{h_{1, \text { num }}\left\lceil X^{\prime}\right.}{h_{1, \text { den }\left\lceil X^{\prime}\right.}}, \ldots, \frac{h_{r, \text { num }}\left\lceil X^{\prime}\right.}{h_{r, \text { den }\left\lceil X^{\prime}\right.}}\right) \\
& =\left(h_{1} \Upsilon_{X^{\prime}}, \ldots, h_{r}\left\lceil_{X^{\prime}}\right)=h \Upsilon_{X^{\prime}},\right.
\end{aligned}
$$

where $h_{i, n u m}^{\prime}, h_{i, d e n}^{\prime} \in A^{\prime}$ and $h_{i, n u m}, h_{i, d e n} \in A$ for $i=1, \ldots, r$, are such that $h_{i}^{\prime}=$ $\frac{h_{i, n u m}^{\prime}}{h_{i, d e n}^{\prime}}$ and $h_{i}=\frac{h_{i, n u m}}{h_{i, \text { den }}}$. The output function $h^{\prime}$ is defined on a $Z$-dense subset of $X^{\prime}$ because $X^{\prime}$ is irreducible and because $h_{i, d e n}\left(x_{0}\right) \neq 0$ for $i=1, \ldots, r$, and thus $h_{i, d e n} \notin I$ for $i=1, \ldots, r$. We define the relation between the rational vector fields $f^{\prime}=\left\{f_{\alpha}^{\prime}: Q^{\prime} \rightarrow Q^{\prime} \mid \alpha \in U\right\}$ and the rational vector fields $f=\left\{f_{\alpha}: Q \rightarrow Q \mid \alpha \in U\right\}$ as

$$
f_{\alpha}^{\prime} \frac{\Psi\left(\left[q_{\text {num }}\right]\right)}{\Psi\left(\left[q_{\text {den }}\right]\right)}=\frac{\Psi\left(\left[\left(f_{\alpha} q\right)_{\text {num }}\right]\right)}{\Psi\left(\left[\left(f_{\alpha} q\right)_{\text {den }}\right]\right)}
$$

for $q=\frac{q_{\text {num }}}{q_{\text {den }}} \in Q$, where $q_{\text {num }}, q_{\text {den }} \in A$ and $q_{\text {den }} \notin I$. Note that we assumed that $\left(f_{\alpha} q\right)_{n u m},\left(f_{\alpha} q\right)_{d e n} \in A$ are such that $f_{\alpha} q=\frac{\left(f_{\alpha} q\right)_{\text {num }}}{\left(f_{\alpha} q\right)_{\text {den }}}$ for a considered $q \in Q$. Since for $\varphi \in A$ such that $\Psi([\varphi])=\varphi^{\prime}$ it is true that $\left.\varphi\right|_{X^{\prime}}=\varphi^{\prime}$, it follows that for $q=\frac{q_{\text {num }}}{q_{\text {den }}} \in Q$, we have that $f_{\alpha}^{\prime} \frac{\Psi\left(\left[q_{n u m}\right]\right)}{\left.\Psi\left(q_{\text {den }}\right]\right)}=\frac{\left(f_{\alpha} q\right)_{n u m}\left\lceil X_{X^{\prime}}\right.}{\left(f_{\alpha} q\right)_{\operatorname{den}}\left\lceil X^{\prime}\right.}=\left(f_{\alpha} q\right)\left\lceil_{X^{\prime}}\right.$, and finally if $q^{\prime}=\frac{\Psi\left(\left[q_{\text {num }}\right]\right)}{\Psi\left(\left[q_{\text {den }}\right]\right)}, q_{\text {den }} \notin I$,

$$
f_{\alpha}^{\prime} q^{\prime}=\left(f_{\alpha} q\right) \Upsilon_{X^{\prime}} .
$$

The well-definedness of the rational vector fields $f_{\alpha}^{\prime}, \alpha \in U$ follows from the fact that $\left(f_{\alpha} \frac{q_{\text {num }}}{q_{\text {den }}}\right)\left\lceil_{X^{\prime}}\right.$ is independent on the choice of representants $q_{\text {num }}, q_{\text {den }} \in A$ of 
classes $\left[q_{\text {num }}\right],\left[q_{d e n}\right] \in A / I$. To verify this, we consider arbitrary $\varphi_{1}, \varphi_{2} \in I$. Thus $\varphi_{1}$ and $\varphi_{2}$ are identically zero on $X^{\prime}$, and therefore $\left(q_{\text {num }}+\varphi_{1}\right)\left\lceil_{X^{\prime}}=q_{\text {num }}\right.$ and $\left(q_{d e n}+\varphi_{2}\right) \uparrow_{X^{\prime}}=q_{d e n}$. Moreover, as $\varphi_{1}=0$ on $X^{\prime}, \varphi_{1}$ is identically zero on the trajectories of the system $\Sigma^{\prime}$, and therefore $\varphi_{1} \circ \tau=0$ on $\widetilde{\mathcal{U}_{p c}}$. Then also $D_{\alpha}\left(\varphi_{1} \circ \tau\right)=0$ on $\widetilde{\mathcal{U}_{p c}}$, and, by Lemma 5.10, $\left(f_{\alpha} \varphi_{1}\right) \circ \tau=0$ on $\widetilde{\mathcal{U}_{p c}}$. Therefore, $f_{\alpha} \varphi_{1}=0$ on $X^{\prime}$, and hence $\left(f_{\alpha} \varphi_{1}\right) \uparrow_{X^{\prime}}=0$. Analogically, $\left(f_{\alpha} \varphi_{2}\right) \uparrow_{X^{\prime}}=0$. Finally, as $f_{\alpha}$ is an $\mathbb{R}$-linear map, $\left(f_{\alpha} \frac{q_{\text {num }}+\varphi_{1}}{q_{\text {den }}+\varphi_{2}}\right) \uparrow_{X^{\prime}}=\left(\frac{\left(f_{\alpha} q_{n u m}+f_{\alpha} \varphi_{1}\right)\left(q_{d e n}+\varphi_{2}\right)-\left(f_{\alpha} q_{d e n}+f_{\alpha} \varphi_{2}\right)\left(q_{n u m}+\varphi_{1}\right)}{\left(q_{d e n}+\varphi_{2}\right)^{2}}\right)$ $\Gamma_{X^{\prime}}=\left(\frac{\left(f_{\alpha} q_{n u m}\right) q_{d e n}-\left(f_{\alpha} q_{d e n}\right) q_{n u m}}{q_{d e n}^{2}}\right) \Gamma_{X^{\prime}}=\left(f_{\alpha} \frac{q_{n u m}}{q_{d e n}}\right) \Gamma_{X^{\prime}}$.

Consider a trajectory $x^{\prime}$ of $\Sigma^{\prime}$ from $x_{0}^{\prime}$ determined by the input $u^{\prime}=\left(\alpha, T_{u^{\prime}}\right) \in$ $\widetilde{\mathcal{U}_{p c}}$ and a trajectory $x$ of $\Sigma$ from $x_{0}$ determined by the input $u=\left(\alpha, T_{u}\right) \in \widetilde{\mathcal{U}_{p c}}$. We assume without loss of generality that $T_{u}=T_{u^{\prime}}$. Then $\frac{d}{d t}\left(\varphi^{\prime} \circ x^{\prime}\right)\left(t ; x_{0}^{\prime}, u^{\prime}\right)=$ $\left(f_{\alpha}^{\prime} \varphi^{\prime}\right)\left(x^{\prime}\left(t ; x_{0}^{\prime}, u^{\prime}\right)\right), \varphi^{\prime}\left(x^{\prime}(0)\right)=x_{0}^{\prime}$ for $t \in\left[0, T_{u}\right], \varphi^{\prime} \in A^{\prime}$, and $\frac{d}{d t}(\varphi \circ x)\left(t ; x_{0}, u\right)=$ $\left(f_{\alpha} \varphi\right)\left(x\left(t ; x_{0}, u\right)\right), \varphi(x(0))=x_{0}$ for $t \in\left[0, T_{u}\right], \varphi \in A$. Since $X^{\prime}=Z$-cl $\left(\mathcal{R}\left(x_{0}\right)\right)$, both trajectories $x$ and $x^{\prime}$ stay in $X^{\prime}$. Let $\varphi^{\prime} \in A^{\prime}$, and let $\varphi \in A$ be such that $\varphi^{\prime}=\Psi([\varphi])$. Because $f_{\alpha}^{\prime} \Psi([\varphi])=\left(f_{\alpha} \varphi\right) \uparrow_{X^{\prime}}, x_{0}=x_{0}^{\prime}$ and because $\varphi=\varphi^{\prime}$ on $X^{\prime}$, it follows that $\left(f_{\alpha} \varphi\right)\left\lceil_{X^{\prime}}\left(x^{\prime}\left(t ; x_{0}^{\prime}, u^{\prime}\right)\right)=\left(f_{\alpha} \varphi\right)\left(x^{\prime}\left(t ; x_{0}^{\prime}, u^{\prime}\right)\right)=\frac{d}{d t}\left(\varphi \circ x^{\prime}\right)\left(t ; x_{0}^{\prime}, u^{\prime}\right)\right.$, and $\varphi\left(x^{\prime}(0)\right)=x_{0}^{\prime}$. Therefore, by Theorem 2.3, the trajectories of the systems $\Sigma$ and $\Sigma^{\prime}$ are the same. Then also the reachable sets of both systems coincide, and thus $X^{\prime}=Z$-cl $\left(\mathcal{R}\left(x_{0}\right)\right)=Z$-cl $\left(\mathcal{R}^{\prime}\left(x_{0}^{\prime}\right)\right)$. So, $\Sigma^{\prime}$ is algebraically reachable.

From the equality of trajectories of $\Sigma$ and $\Sigma^{\prime}$ and from the definition of the output function $h^{\prime}$ of $\Sigma^{\prime}$, it follows that the system $\Sigma^{\prime}$ is a rational realization of $p$. More precisely, let $u \in \widetilde{\mathcal{U}_{p c}}$; then

$$
\begin{aligned}
p(u) & \left.=h\left(x\left(T_{u} ; x_{0}, u\right)\right) \text { (because } \Sigma \text { realizes } p\right) \\
& \left.=\left(h \Upsilon_{X^{\prime}}\right)\left(x\left(T_{u} ; x_{0}, u\right)\right) \text { (because } Z \text {-cl }\left(\mathcal{R}\left(x_{0}\right)\right)=X^{\prime}\right), \\
& \left.=\left(h \Upsilon_{X^{\prime}}\right)\left(x^{\prime}\left(T_{u} ; x_{0}^{\prime}, u\right)\right) \text { (from the equalities of the trajectories of } \Sigma \text { and } \Sigma^{\prime}\right), \\
& \left.=h^{\prime}\left(x^{\prime}\left(T_{u} ; x_{0}^{\prime}, u\right)\right) \text { (by the definition of } h^{\prime}\right) .
\end{aligned}
$$

Thus, the system $\Sigma^{\prime}$ is an algebraically reachable rational realization of $p$. We prove that $\Sigma^{\prime}$ is also rationally observable. Let us compute the observation field $Q_{o b s}\left(\Sigma^{\prime}\right)$ of the system $\Sigma^{\prime}$. First, the observation algebra $A_{o b s}\left(\Sigma^{\prime}\right)$ is the smallest algebra containing the elements $h_{i}^{\prime}, f_{\alpha}^{\prime} h_{i}^{\prime}$ for $i=1, \ldots, r$, and $\alpha=\left(\alpha_{1}, \ldots, \alpha_{k}\right)$ such that $k \in \mathbb{N}, \alpha_{j} \in U, j=1, \ldots k$. As $h_{i}^{\prime}=h_{i}\left\lceil_{X^{\prime}}\right.$ and $f_{\alpha}^{\prime} h_{i}^{\prime}=f_{\alpha} h_{i}\left\lceil_{X^{\prime}}\right.$ for $i=1, \ldots, k$, and $\alpha=\left(\alpha_{1}, \ldots, \alpha_{k}\right), \alpha_{j} \in U, k \in \mathbb{N}$, we get that $\varphi=\frac{\varphi_{\text {num }}}{\varphi_{\text {den }}} \in A_{\text {obs }}(\Sigma)$, where $\varphi_{\text {num }}, \varphi_{\text {den }} \in A$ if and only if $\varphi^{\prime}=\frac{\Psi\left(\left[\varphi_{\text {num }}\right]\right)}{\Psi\left(\left[\varphi_{\text {den }}\right]\right)} \in A_{\text {obs }}\left(\Sigma^{\prime}\right)$. Furthermore, since $A_{\text {obs }}(\Sigma)$ and $A_{o b s}\left(\Sigma^{\prime}\right)$ are integral domains, $\varphi=\frac{\varphi_{\text {num }}}{\varphi_{\text {den }}} \in Q_{\text {obs }}(\Sigma), \varphi_{\text {den }} \notin I$ if and only if $\varphi^{\prime}=\frac{\Psi\left(\left[\varphi_{\text {num }}\right]\right)}{\left.\Psi\left(\varphi_{\text {den }}\right]\right)} \in Q_{\text {obs }}\left(\Sigma^{\prime}\right)$. Because the system $\Sigma$ is rationally observable and because $\mathcal{Q}(\Psi(A / I)) \cong \mathcal{Q}\left(A^{\prime}\right)$, we derive that the system $\Sigma^{\prime}$ is also rationally observable.

(ii) $\Rightarrow$ (iii) We assume that $p$ has a canonical rational realization $\Sigma=\left(X, f, h, x_{0}\right)$. From Theorem 5.16, $Q_{o b s}(p)$ is finitely generated.

(iii) $\Rightarrow$ (i) We assume that the observation field $Q_{o b s}(p)$ of $p$ is finitely generated. From Proposition 5.13, $Q_{o b s}(p)$ is finitely generated by the elements of $\mathcal{A}\left(\widetilde{\mathcal{U}_{p c}} \rightarrow \mathbb{R}\right)$. Let $Q_{o b s}(p)=\mathbb{R}\left(\varphi_{1}, \ldots, \varphi_{k}\right)$, where $\varphi_{i} \in \mathcal{A}\left(\widetilde{\mathcal{U}_{p c}} \rightarrow \mathbb{R}\right), i=1, \ldots, k$. By the definition of observation field, $Q_{o b s}(p)$ is closed with respect to $D_{\alpha}$ derivations $\alpha=\left(\alpha_{1}, \ldots, \alpha_{i}\right), i \in \mathbb{N}, \alpha_{j} \in U, j=1, \ldots, i$. To prove that there exists a rationally observable rational realization of $p$, we construct a rational realization $\Sigma=\left(X, f, h, x_{0}\right)$ of $p$ such that $Q_{o b s}(\Sigma)=Q$, where $Q$ denotes the field of rational functions on $X$. 
The field $F=Q_{o b s}(p)=\mathbb{R}\left(\varphi_{1}, \ldots, \varphi_{k}\right)$ fulfills the conditions (i)-(iii) of Proposition 5.14. By following the proof of Proposition 5.14 with $F=Q_{o b s}(p)$, we construct a rational realization $\Sigma=\left(X, f, h, x_{0}\right)$ of $p$ as

$$
\begin{aligned}
X & =\mathbb{R}^{k}, \\
f_{\alpha} & =\sum_{i=1}^{k} v_{i}^{\alpha}\left(X_{1}, \ldots, X_{k}\right) \frac{\partial}{\partial X_{i}}, \quad \alpha \in U, \\
h_{j}\left(X_{1}, \ldots, X_{k}\right) & =w_{j}\left(X_{1}, \ldots, X_{k}\right), \quad j=1 \ldots r, \\
x_{0} & =\left(\varphi_{1}(e), \ldots, \varphi_{k}(e)\right) .
\end{aligned}
$$

This realization is such that

$$
\begin{aligned}
p_{j} & =w_{j}\left(\varphi_{1}, \ldots, \varphi_{k}\right), \quad j=1 \ldots r, \text { and } \\
D_{\alpha} \varphi_{i} & =v_{i}^{\alpha}\left(\varphi_{1}, \ldots, \varphi_{k}\right), i=1 \ldots k .
\end{aligned}
$$

Because $X=\mathbb{R}^{k}$, we get for the field $Q$ of rational functions on $X$ that $Q=$ $\mathbb{R}\left(X_{1}, \ldots, X_{k}\right)$. To consider $h_{j}$ and $f_{\alpha} h_{j}, j=1 \ldots r$, is the same as to consider $p_{j}$ and $D_{\alpha} p_{j}$ but in different coordinates. Therefore, $Q_{o b s}(\Sigma)$, as a field of quotients of the smallest subalgebra of $Q$ containing all $h_{j}, f_{\alpha} h_{j}$, where $j=1, \ldots, r, \alpha=$ $\left(\alpha_{1}, \ldots, \alpha_{k}\right), k \in \mathbb{N}, \alpha_{i} \in U, i=1, \ldots, k$, equals $\mathbb{R}\left(X_{1}, \ldots, X_{k}\right)$ in analogy to the relation $Q_{o b s}(p)=\mathbb{R}\left(\varphi_{1}, \ldots, \varphi_{k}\right)$. That means that $Q_{o b s}(\Sigma)=\mathbb{R}\left(X_{1}, \ldots, X_{k}\right)=Q$, which proves the rational observability of $\Sigma$.

Corollary 6.2. Let $p$ be a response map. According to Theorem 5.16 and Theorem 6.1, the following statements are equivalent:

(i) $p$ is realizable by a rational system;

(ii) $p$ has a rational realization which is rationally observable;

(iii) $p$ has a rational realization which is canonical; thus both rationally observable and algebraically reachable.

7. Concluding remarks. We have provided sufficient and necessary conditions for a response map to be realizable by a rational system. Since the proof of Proposition 5.14 concerning sufficient conditions for the existence of a rational realization for a given response map is constructive, it provides an algorithm for constructing a rational system realizing a response map. We proved that for a given response map the problems of the existence of a rational/rationally observable rational/canonical rational realization are equivalent. The proof of Theorem 6.1 stating this equivalence is also constructive. It provides an algorithm for the construction of a canonical rational realization from a rationally observable rational realization. Moreover, it shows that if we consider as a field $F$ from Proposition 5.14 the observation field of a response map to be realized, then the algorithm for constructing a rational realization from Proposition 5.14 gives as a result a rationally observable rational realization. Additional results which we got by studying minimal rational realization can be found in [15]. For some remarks on algebraic reachability of rational systems, see [14].

We work with irreducible real affine varieties for two reasons. First, irreducibility simplifies the technical details of the proofs. Second, working with the real varieties allows us to have a better geometric understanding of the state-spaces of rational systems, and it is also sufficient for applications, for example, in systems biology. It is possible to generalize the results of this paper for reducible varieties.

Algebraic framework we use can be useful from a computational point of view. The procedures to check the properties of rational systems such as rational observability, algebraic reachability, and minimality of rational realizations are formulated 
in [15]. They can be implemented by using already existing computer algebra packages. The procedures for constructing rational realizations of desired properties are still to be developed.

Further research is required on the realization theory for rational positive systems. There are several issues to be overcome in that research.

Acknowledgment. The authors are very grateful to Mihály Petreczky for long discussions, useful comments, and advice.

\section{REFERENCES}

[1] Z. Bartosiewicz, Realizations of polynomial systems, in Algebraic and Geometric Methods in Nonlinear Control Theory, M. Fliess and M. Hazenwinkel, eds., D. Reidel, Dordrecht, 1986, pp. 45-54.

[2] Z. Bartosiewicz, Ordinary differential equations on real affine varieties, Bull. Pol. Acad. Sci. Math., 35 (1987), pp. 13-18.

[3] Z. Bartosiewicz, Rational systems and observation fields, Systems Control Lett., 9 (1987), pp. 379-386.

[4] Z. Bartosiewicz, Minimal polynomial realizations, Math. Control Signals Systems, 1 (1988), pp. 227-237.

[5] N. Bourbaki, Algebra II: Chapters 4-7, Elem. Math. (Berlin), Springer-Verlag, Berlin, 1990.

[6] C.I. Byrnes and P.L. FAlb, Applications of algebraic geometry in system theory, Amer. J. Math., 101 (1979), pp. 337-363.

[7] D. Cox, J. Little, And D. O'Shea, Ideals, Varieties, and Algorithms: An Introduction to Computational Algebraic Geometry and Commutative Algebra, 3rd ed., Springer-Verlag, Berlin, 2007.

[8] P. FALB, Methods of Algebraic Geometry in Control Theory: Part 1, Scalar Linear Systems and Affine Algebraic Geometry, Birkhäuser, Boston, 1990.

[9] P. FALB, Methods of Algebraic Geometry in Control Theory: Part 2, Multivariable Linear Systems and Projective Algebraic Geometry, Birkhäuser, Boston, 1999.

[10] N. Jacobson, Basic Algebra II, W. H. Freeman, San Francisco, 1980.

[11] B. JAKUBCZYK, Existence and uniqueness of realizations of nonlinear systems, SIAM J. Control Optim., 18 (1980), pp. 455-471.

[12] E. KunZ, Introduction to Commutative Algebra and Algebraic Geometry, Birkhäuser, Boston, 1985.

[13] S. LANG, Algebra, Addison-Wesley, Reading, MA, 1965.

[14] J. NĚmcovÁ, Algebraic reachability of rational systems, in Proceedings of European Control Conference, Budapest, Hungary, 2009.

[15] J. NĚmcová And J.H. van Schuppen, Realization theory for rational systems: Minimal rational realizations, Acta Appl. Math., to appear.

[16] E.D. Sontag, Polynomial Response Maps, Lecture Notes in Control and Inform. Sci. 13, Springer-Verlag, Berlin, Heidelberg, 1979.

[17] J.H. VAN SCHUPPEN, System theory of rational positive systems for cell reaction networks, in Proceedings of the International Symposium of Mathematical Theory of Networks and Systems, Leuven, Belgium, 2004.

[18] Y. WANG AND E.D. SontAG, Algebraic differential equations and rational control systems, SIAM J. Control Optim., 30 (1992), pp. 1126-1149.

[19] D.J. Winter, The Structure of Fields, Springer-Verlag, New York, 1974.

[20] O. Zariski and P. Samuel, Commutative Algebra I, II, Springer-Verlag, Berlin, 1958.

Copyright $@$ by SIAM. Unauthorized reproduction of this article is prohibited. 